\title{
Italique
}

Poésie italienne de la Renaissance

VII | 2004

Varia

\section{Il volo, il desiderio, la caduta : Icaro Nella Lirica italiana efrancese del XVI secolo}

Stefano Prandi

\section{(2) OpenEdition}

Edizione digitale

URL: https://journals.openedition.org/italique/130

DOI: $10.4000 /$ italique. 130

ISSN: 1663-4438

\section{Editore}

Librairie Droz

\section{Edizione cartacea}

Data di pubblicazione: 1 novembre 2004

Paginazione: 101-135

ISBN: 2-600-00976-0

ISSN: 1423-3983

\section{Notizia bibliografica digitale}

Stefano Prandi, «Il volo, il desiderio, la caduta : Icaro Nella Lirica italiana efrancese del XVI secolo», Italique [Online], VII | 2004, online dal 05 octobre 2009, consultato il 21 septembre 2021. URL: http:// journals.openedition.org/italique/130 ; DOI: https://doi.org/10.4000/italique.130

(c) Tous droits réservés 


$$
\begin{aligned}
& \text { Stefano PRANDi } \\
& \text { I L VOLO, IL DESIDERIO, } \\
& \text { L A C A D T A : } \\
& \text { I C A R O NELLA LIRICA ITALIA N A } \\
& \text { E FRANCESE DEL XVI SECOLO }
\end{aligned}
$$




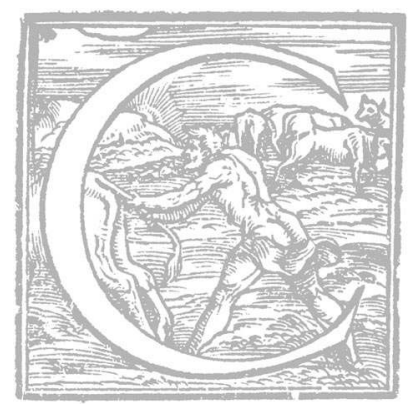

Ifra stessa dell'oltranza propria dell'attività letteraria, il mito icario misura nella figura della caduta quella «nostalgie inexpiable de la hauteur» che Bachelard vede come caratteristica dell'immaginazione dinamica rappresentata nel motivo poetico del volo. ${ }^{\mathrm{I}}$ La vicenda di Icaro appare connotata da una circolarità che conosce un movimento dal basso verso l'alto, marcato nello stesso tempo in senso positivo e negativo dalla hybris che lo caratterizza -: l'audacia, la tensione verso la conoscenza che si fa desiderio da un lato, e dall'altro, la negazione della virtù classica della metriotes -; e da un complementare movimento dall'alto verso il basso, la caduta, a seguito del fatale appressamento al sole. Un vettore orientato in senso verticale caratterizza dunque in senso esclusivo il racconto icario, con un orientamento simbolico che vede la progressione ascendente legata al fuoco $e$ alla luce (il sole), e quella discendente all'acqua $e$ alle tenebre (il mare, tomba di Icaro). É proprio questo particolare che costituisce un tratto di forte similarità tra l'episodio di Icaro e quello di Fetonte, anch'egli incapace di mantenere il «giusto mezzo» nel suo temerario volo, punito egualmente col fuoco (il fulmine di Giove), e conclusosi nelle acque del fume Po. Se si tiene conto della scala gerarchica degli elementi nel mondo antico, che vede la progressione in senso ascendente di dignità (terra, acqua, aria, fuoco) si può notare come in entrambi $i$ miti l'abbandono dell'elemento più basso (terra), compiuto per aspirare ad un grado superiore (lo spazio aereo), venga punito, per mezzo dell'elemento in assoluto più nobile (fuoco), con una sorta di declassamento di stato: dalla sfera elementare dell'aria a quella dell'acqua. L'enfasi sulle conseguenze negative e dolorose dell'aspirazione icaria alle celesti altezze costituisce un aspetto essenziale del mito. La punizione della hybris per il mancato rispetto del giusto mezzo è il suo esplicito, quasi imbarazzante contenuto morale che si offre, come tema ineludibile, alla poesia antica, la quale reagisce alla sollecitazione con l'arma della variatio o dell'oltranza stilistica o tematica per tentare di salvaguardare la complessità e l'ambiguità del linguaggio letterario, minacciata dall'invadenza didascalica del mito.

\section{Altius egit iter: l'età classica}

Forse la testimonianza più eloquente di quanto si va dicendo è il passo dell'Eneide che descrive l'approdo dei Troiani sulle coste di Cuma e il mirabile portale del tempio di Apollo; l'ecfrasis, realizzata attraverso il 
medium dello sguardo di Enea, che si sdipana lungo il filo rosso delle imprese di Dedalo:

\section{[...] Tu quoque magnam}

partem opere in tanto, sineret dolor, Icare, haberes.

Bis conatus erat casus effingere in auro;

bis patriae cecidere manus. [...]

$$
\text { (Aen. VI, 30-33) }
$$

Un eccesso improvviso di pathos sembra insorgere a questo punto della visione, una straordinaria rappresentazione in progress di non finito, apice emozionale che interrompe il lavoro dell'artista Dedalo e pare comunicarsi ad Enea stesso (infine, per suo tramite, al lettore). Silenzio e spazio vuoto, un'assenza assai piu eloquente di qualunque altro artistico conato, sono le uniche due risposte possibili all'oltranza a cui il mito icario qui allude: con esso si conclude infatti l'ecfrasis del portale, prima che la sibilla e Acate raggiungano Enea e $i$ suoi.

Nelle Metamorfosi di Ovidio, poema della variazione per eccellenza, ritroviamo la vicenda di Icaro nella sua versione più diffusa e circostanziata. La strategia ovidiana appare ben chiara: discolpare il protagonista, mitigare il nucleo bruciante della hybris. Il «puer Icarus», ignaro della minaccia che lo sourasta (Met. VIII I96), gioca con lo strumento che causerà la sua rovina, con una capricciosa spensieratezza che, ostacolando l'ingegnosa attività paterna, cela quasi una inconsapevole precognizione degli eventi futuri:

\section{[...] Puer Icarus una \\ stabat et, ignarus sua se tractare pericla, ore renidenti modo quas vaga moverat aura captabat plumas, flavam modo pollice ceram mollibat lusuque suo mirabile patris impediebat opus $[\ldots]$}

(Met. VIII, I95-200)

Dedalo appare d'altra parte consapevole dei rischi della sua sfida alla natura («[...] ignotas animum dimittit in artes / naturamque novat» I88-89; «[...] damnosas erudit artes» 2I5; "devovitque suas artes» 234), e preoccupato per l'inesperienza del figlio, che ammonisce ("[...] "Medio [...] ut limite curras, / Icare", ait "moneo, ne, si demissior ibis, / unda gravet pennas, si celsior, ignis adurat"》) non senza cedere al pianto ("Inter opus monitusque genae maduere seniles / et patriae tremuere manus [...]»). Nonostante $i$ consigli paterni, il destino di Icaro ben presto si compie nel segno dell'infrazione di quella 
medietas che era stata indicata come unica possibilità di salvezza: «coeli cupidine tractus, altius egit iter» (Met. VIII, 224-25).

Anche nella seconda versione ovidiana del mito, ${ }^{2}$ Ars amandi II, I5-98, Dedalo viene presentato come un uomo senza scampo, ${ }^{3}$ per nulla blasfemo - come rivela l'invocazione a Giove dei vv. 38-40 - costretto a ricorrere al suo ingegno per raggiungere la patria amata. La metafora del volo, analogamente alle Metamorfosi, è associata in prevalenza alle immagini nautiche: le ali sono chiamate «remigium volucrum» (4); cfr. Met. VIII, 228 «remigioque carens non ullas percipit auras»; s: "His patria est adeunda carinis»). Ritorna il medesimo sintagma altius egit iter, ma preceduto da una puntualizzazione che vale come correzione di un possibile risvolto eroico del personaggio: «incautis nimius temerarius annis» (83). Ovidio sembra dunque risolvere la straordinaria tensione gnomica del mito con una strategia narrativa mirante a sottolineare la fragilità di Icaro, a intensificare gli effetti patetici del racconto della caduta, osservata dallo specola del padre. L'epilogo della vicenda icaria riprende volutamente quello di Fetonte, a cui però è riservato l'onore di una tomba con tanto di epigrafe:

Naides Hesperiae trifida fumantia flamma corpora dant tumulo, signant quoque carmine saxum: «Hic situs est Phaëthon, currus auriga paterni, quem si non tenuit, magnis tamen excidit ausis »;

(Met. II, 325-28)

mentre nell'episodio di Icaro spicca il contrasto tra l'assenza di un qualsiasi sepolcro e la vastità della fama conquistata con la caduta, che ha potuto dare il nome ad un mare: «Ossa tegit tellus, aequora nomen habent» (Ars am. II, 96); "Icarus immensas nomine signet aquas» (Tristia III, 22). Questa straordinaria capacita di conservare perennemente, attraverso la nominazione, la memoria di un'impresa degna di nota, avvicina l'epilogo della vicenda di Icaro agli esiti propri della scrittura letteraria: precisamente ciò che troviamo nell'elaborazione del topos da parte di Orazio.

Orazio è forse, tra gli autori latini, il più convinto assertore della necessità dell'aurea mediocritas ma anche uno degli scrittori con la più profonda coscienza del valore insostituibile che assume la missione del poeta; ed è proprio nella celebre ode XX del secondo libro, che descrive la metamorfosi dell'autore in cigno, animale sacro ad Apollo, che ritroviamo in filigrana, come controcanto negativo, l'esempio icario:

Non usitata nec tenui ferar pinna biformis per liquidum aethera vates neque in terris morabor longius invidiaque maior 


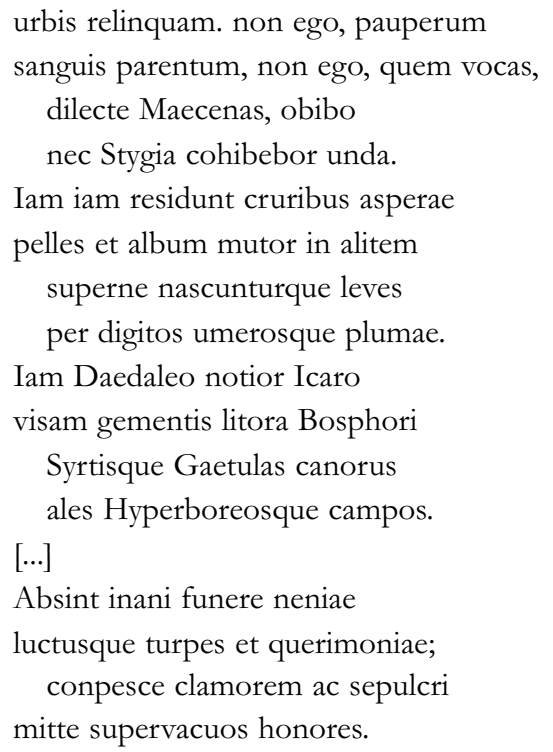

$(O d \text {. II, Xx, I-I 6, ) })^{4}$

La metamorfosi e il volo adombrano un rischio, la possibilità di cadere in acque mortali, proprio come quelle icarie («Non ego [...] obibo / nec Stygia cobibebar unda»); nemmeno Orazio, infine, resterà prigioniero di un sepolcro - realtà che anche Icaro non aveva conosciuto - poichè la poesia lo strapperà al limitato destino dell'uomo comune: la tomba sarà inanis, 'le ali della fama avranno già condotto in altissime regioni ciò che resta del poeta. Sembra dunque pienamente accettabile la lezione, al v. I3, notior Icaro, che nella tradizione manoscritta si contrappone a ocior Icaro; ${ }^{7}$ nonostante la caduta, l'audace figlio di Dedalo ba saputo raggiungere fama imperitura, come Orazio riconosce, benché poi ritagli per sé una condiz̨ione ancora migliore. ${ }^{8} \dot{E}$ dunque attraverso una contaminazione a livello tematico (nella quale il motivo icario viene a fondersi con quello del cigno) che Orazio tenta di neutralizzare la minacciosa cogenza simbolica del mito, la sua imperativa prescrittività.

L'ultimo autore che ci permette di concludere questo rapido abbozzo sulle tipologie di "risposta" che la tradizione latina realizza su sollecitazione del mito icario (tipologie che, come vedremo, si dimostrano fondamentali per comprendere gli sviluppi lirici del tema in ambito volgare), è Seneca tragico, $e$ in particolare nei vv. 675-9I dell' Hercules Oeteus:

Quisquis medium defugit iter stabili numquam tramite currit: dum petit unum praebere diem patrioque puer constitit axe 
nec per solitum decurrit iter, sed Phoebeis ignota petens sidera flammis errante rota, secum pariter perdidit orbem. medium caeli dum sulcat iter, tenuit placitas Deadalus oras nullique dedit nomina ponto; sed dum volucres vincere veras Icarus audet patriasque puer despicit alas Phoeboque volat proxumus ipsi, dedit ignoto nomina ponto: male pensantur magna ruinis.

(Hercules Oeteus, 675-9I)

Come ha mostrato Giuseppe Velli, in un saggio dedicato al sonetto LXXIX del Sannazaro, ${ }^{9}$ i versi senecani costituiscono un vero palinsesto del libro II ed VIII delle Metamorfosi di Ovidio, ${ }^{\text {IO }}$ rispettivamente dedicati a Fetonte ed Icaro, oltre che di altri luoghi paralleli dell'Ars amandi ${ }^{\mathrm{II}}$ e dei Tristia. ${ }^{\mathrm{I}}$ Seneca prende in carico senza esitazioni il contenuto morale del mito, rivelando un approccio decisamente orientato verso la condanna dell'eccessiva audacia dei due temerari eroi: ${ }^{3}$ le sententiae dei $v v$. 675-76 e 69I incorniciano con assoluta regolarità una esemplarità negativa, costituita da Fetonte ( $c f r$. i vv. 677-82, che ad esso alludono) e dallo stesso Icaro (686-90), dittico che a sua volta funge da cornice all'unico esempio virtuoso, quello di Dedalo, che si trova valorizzato nella parte centrale del componimento (683-85): una medietas strutturale che allude alla virtù che ha permesso all'artifex di sopravvivere. Al quadro delineato da Velli bisogna tuttavia aggiungere un altro testo di Seneca, l'Oedipus, che ai vv. 892-9II recita:

Gnosium regem timens astra dum demens petit artibus fisus novis certat et veras aves vincere ac falsis nimis imperat pinnis puer, nomen eripuit freto. Callidus medium senex Daedalus librans iter nube sub media stetit alitem expectans suum (qualis accipitris minas 


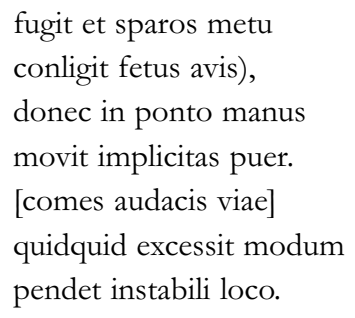

(Oedipus, 892-91 I)

Ritorna la formula narrativa dum [...] petit (893; cfr. Herc. Oet. $677 e$ Ovidio, Trist. I, I, 88), ritorna, ma caricata di un significato di valore opposto rispetto all ipotesto ovidiano, la parola-chiave ars: artibus fisus novis (895) capovolge l'Icarus audaci fortius arte volat $d i$ Ars am. $I, 76^{14}$ (novae [...] labor artis comunque connota la laboriosa fabbricazione delle ali dei due personaggi, Ars am. II, 48); d'altra parte, se per Ovidio Icaro è incautis nimium temerarius annis (Ars am. I, 83), per Seneca egli è soltanto demens (Oed. 893). V'è infine il particolare su cui più insiste il sonetto sannazariano, quello del nomen (LXXIX, I2), della fama raggiunta da Icaro per la sua audace impresa; l'Oedipus presenta qui significativamente nomen eripuit freto ( $898 ;$ cfr. Herc. Oet. 689-90), a fronte di una serie massiccia di qualificazioni positive, o tutt'al più neutre, di Ovidio. ${ }^{15}$ L'accoglimento della valenza etico-simbolica del mito si traduce in Seneca in una sorta di tour de force a livello elocutivo (con esiti spesso brachilogici, come hanno sottolineato gli interpreti) e intertestuale, quasi a voler chiudere $i$ conti con un'intera tradizione. I due brani citati, in effetti, si presentano quasi come centoni: oltre ai rilievi già fatti, si aggiunga, ad esempio, che «patriaque puer constitit axe» (Herc. Oet. 677), richiama Met. II, 59-60 «Non tamen ignifero quisquam consistere in axe», e che l'immagine della competizione di Icaro con gli uccelli dei vv. 896-97 riprende Met. VIII, I94-95. Seneca infine riveste un ruolo importante, in rapporto ai futuri sviluppi poetici del mito, oltre che per la sua funzione compendiaria, anche per aver esplicitamente congiunto le vicende di Icaro e Fetonte in un unico campo di esemplarità: aspetto di cui terrà conto tutta la tradizione successiva.

V'è naturalmente un'ulteriore, fondamentale risposta che la letteratura oppone agli aspetti più inquietanti del mito, anche se, dal nostro punto di vista, essa assume un valore secondario: quella che consiste negli esiti satirico-parodistici: è il caso della terza satira di Giovenale, nella quale la figura di Icaro è allusa per rappresentare ironicamente l'invadenza e la saccenteria dei greci romanizzati, capaci persino di spiccare il volo, se lo si chiede loro: ${ }^{16}$ o ancora l'Icaromenippo di Luciano, in cui il volo di conoscenza del protagonista disvela semplicemente tutte le meschinità della vita umana. 
Un importante sviluppo che esula invece dagli aspetti satirici appena ricordati si ritrova nello pseudo-lucianeo De astrologia: nell'operetta si sostiene che Dedalo trasmise colpevolmente al figlio i segreti dell'astrologia; questi sollevò temerariamente la propria mente al cielo, e fini per precipitare nel mare dell'errore. ${ }^{17}$ Tale interpretazione, che si ritrova nel commento di Eustazio a Iliade II, I45, sarà ripresa in un celebre passaggio delle Disputationes adversus astrologiam divinatricem del Pico (III, 9):

Qui enim Homerum allegorice exponunt, per illam Icari fabulam, qui facticiis pennis caelo tenuiore se committens in mare precipitatus est, astrologos aiunt designari, qui pennis temerariae professionis in caelum se substollentes, cum de coelestibus suis dogmatis, quibus sublimari videntur, quid sint praedicturi, in pelagus ruunt mendaciorum. ${ }^{\text {I } 8}$

Lo spunto sarà poi consegnato ad una fama europea dall'emblema I04 di Alciati (imitatio dell'epigramma CVII dell'Appendix planudea dell'Anthologia palatina), che rappresenta appunto il momento drammatico della caduta, ed è accompagnato dal seguente epigramma:

Icare, per superos qui raptus et aera, donec

In mare praecipitem cera liquata daret,

Nunc te cera eadem, fervensque resuscitat ignis,

Exemplo ut doceas dogmata certa tuo.

Astrologus caveat quicquam praedicere: praeceps

Nam cadet impostor dum super astra volat. ${ }^{\text {I9 }}$

Tra $i$ tanti esempi dell'ampia fortuna di questa esegesi allegorica possiamo annoverare Pontus de Tyard; che nella Mantice. Discours de la verité de divination par astrologie interpreta la figura di Icaro come «le judiciaire prosumptueux» e quella di Dedalo come «le modeste philosophe naturel ou mathematicien tout formé de raison». ${ }^{20}$

\section{Icarus redivivus: la tradizione volgare}

Nelle ultime pagine siamo già trascorsi a quel processo di consolidamento dell'allegoresi che caratterizza la tradizione interpretativa medievale. Naturalmente si assiste ad una radicale risemantizzazione del significato complessivo del mito: cosi, in un'opera tarda come l'Ovide moralisé (XIV sec.), la metriotes espressa dalla traettoria mediana del volo diviene equilibrio armonioso tra l'ala destra (l'amore di Dio) e quella sinistra (l'amore del prossimo), cosi da evitare la colpevole «outrecuidance». ${ }^{2 \mathrm{I}}$ Diviene inoltre assai comune 
l'accostamento di Icaro a Fetonte, ad illustrare una comune esemplarità negativa: ad esempio in Giovanni di Salisbury. ${ }^{22}$ Tale aspetto si ritrova anche nella Commedia, che avvale dell'esempio per esprimere la vertigine provata da Dante durante il volo in groppa a Gerione, tra il settimo e l'ottavo cerchio:

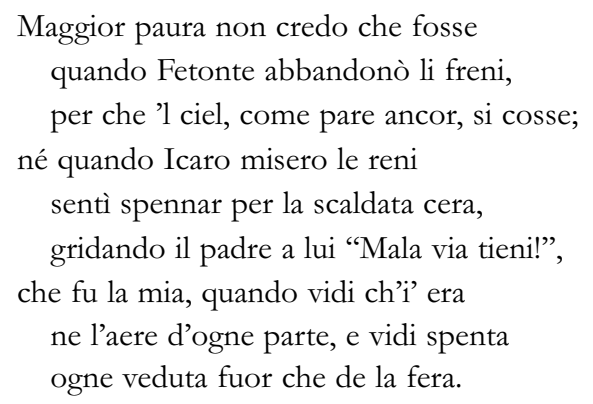

(Inf. 17, I06-14)

Significativamente, le domande concitate che, in Ovidio, Dedalo rivolge al figlio $^{23}$ divengono qui, forse per influenza secolare di integumenta esplicitamente orientati in senso morale, quasi un monito severo. La sintesi potente offerta dal passo dantesco influenzerà il Boccaccio, che nel Filocolo ne riprodurrà le movenze $;^{24}$ è soprattutto l'aggettivo dantesco misero che rimane impresso nell'immaginazione del certaldese, sempre incline alla «compassione degli afflitti): oltre che nel Filocolo ritroviamo il termine nella Commedia delle ninfe e nell'Elegia di Madonna Fiammetta. ${ }^{25}$

All'interno del Canzoniere petrarchesco l'immagine del volo qualifica solitamente la dinamica del desiderio (Rvf. I77, I-4, ${ }^{26}$ Rvf. I82, I2-I4) ${ }^{27}$ oppure dell'immaginazione poetica; quest'ultima accezione conosce forse gli sviluppi più consistenti: vedi almeno 302, I-2: "Levommi il mio penser in parte ov'era / quella ch'io cerco, et non ritrovo in terra» e 362, $I$ : "Volo con l'ali de' pensieri al cielo». Malgrado i riferimenti sparsi non arrivino a configurare, in rapporto al mito di Icaro, una vicenda unitaria, soprattutto importante appare in questo contesto il sonetto 307:

I' pensava assai destro esser su l'ale, non per lor forza, ma di chi le spiega, per gir cantando a quel bel nodo eguale onde Morte m'assolve, Amor mi lega.

Trovaimi a l'opra via più lento et frale d'un picciol ramo cui gran fascio piega, et dissi: - A cader va chi troppo sale, né si fa ben per uom quel che 'l ciel nega. - 
Mai non poria volar penna d'ingegno, nonché stil grave o lingua, ove Natura volò, tessendo il mio dolce ritegno.

Seguilla Amor con sì mirabil cura in adornarlo, ch'i' non era degno pur de la vista: ma fu mia ventura.

(Rvf. 307)

Risulta evidente, in primo luogo, come il poeta si avvalga dell'ambiguità semantica del termine penna, nella quale risulta compendiata sia l'idea del volo fisico, sia le risorse offerte all'immaginazione dalla scrittura letteraria. ${ }^{28}$ Forse non sarà un caso il fatto che si possa registrare proprio in questo contesto il verso sentenzioso «a cader va chi troppo sale», sorta di replica inappellabile all'ovidiano «magnis tamen excidit ausis» (Met. II, 328). Benché la figura di Icaro non venga evocata esplicitamente, sembra di intravedere in questa risoluzione perentoria, in filigrana, come nel caso dantesco poc'anzi discusso, quasi gli effetti della cogenza morale del mito. L'ammissione di infirmitas del Petrarca, il suo mantenere a distanza la hybris dichiandosi inferiore alla natura, impediscono che egli condivida il destino icario di una rovinosa caduta: e il sonetto può chiudersi cosi nel segno della «ventura». Va poi tenuto in conto che nei Rerum vulgarium fragmenta l'immagine del volo assume anche un significato diverso, quasi antitetico a quello classico dell'oltranza del desiderio o della conoscenza, soprattutto per influenza del Salmo 54, 7 "Qui dabit mihi pennas sicut columbae / et volabo et requiescam?», come mostra:

Qual gratia, qual amore, o qual destino mi darà penne in guisa di colomba, ch'i' mi riposi, et levimi da terra?

$$
(R v f .8 \mathrm{I}, \mathrm{I} 2-\mathrm{I} 4)^{29}
$$

Un momento di ulteriore elaborazione del tema ̀̀ costituito dall'esegesi condotta dal neoplatonismo fiorentino ${ }^{30}$ sul passo del Fedro (25 I C sgg) che descrive la fenomenologia amorosa come una condizione nella quale l'anima mette le ali; il desiderio della bellezza, prima fonte dell'innamoramento, innesca cosi un processo nel quale gli individui vengono a trovarsi in un «circulo» amoroso che da Dio procede verso le creature ed a lui ritorna. ${ }^{3 \mathrm{I}}$ In un quadro simile, è evidente che il volo del desiderio, che la letteratura rinascimentale rappresenterà anche attraverso la figura di Icaro, perda $i$ suoi connotati negativi sul piano morale e assuma, rispetto alle interpretazioni allegoriche medievali del mito, un valore diametralmente opposto a livello apotelesmatico: non più fuga dalla verità, tentata sull'onda di una folle presunzione, ma approssimazione ad essa. 
Su questo sfondo reagiscono le componenti petrarchistiche del primo Cinquecento, in un quadro dottrinale fatalmente semplificato; per una precoce attestazione si legga ad esempio Equicola, Libro di natura de amore I, I e Bembo, Asolani I, XXV:

Non è causa di mestitia amore, ma advene questo per colpa de homini di amare et farse amare imperiti. Dice Aristotele che ad torto multe volte li amatori se lamentano che, amando essi vehementemente, non siano con mutuo amore reamati, non essendo in loro cosa amabile. Se noi noi medesmi cognoscessemo, non tentaremo ascendere sopra nostra conditione; ma volendo più alto che le forze non supportano volare, Icari et miserabili Phaetonti ci retrovamo; nel che nostra imprudentia, non amor si deve accusare. Il quale se dolce amaro il poeta Orpheo nomina, se di fele et mele fecundissimo Plauto lo existima, se dolce amaro parimente pigliare lo amante Apuleio referisce, se Venere misticare col dolce lo amaro Catullo canta, se doi fiumi, l'un dolce, l'altro amaro, neli horti di Venere Claudiano finge, non è senza causa, che multe volte, per dubii et periculosi scogli lo amante al desiderato porto è necessitato il curso tendere, et da incerte speranze et varii timori sollicitato, di naufragio è constrecto dubitare, donde nascono tanti stridi deli miseri amanti.

O amara dolcezza, o venenata medicina de gli amanti non sani, o allegrezza dolorosa, la qual di te nessun più dolce frutto lasci a' tuoi possessori che il pentirsi; o vaghezza che, come fumo lieve, non prima sei veduta che sparisci, né altro di te rimane ne gli occhi nostri che il piagnere; $\mathrm{o}$ ali che bene in alto ci levate perché, strutta dal sole la vostra cera, noi con gli homeri nudi rimanendo, quasi novelli Icari, cadiamo nel mare. Cotali sono i piaceri, donne, i quali amando si sentono.

Ma veniamo finalmente alla lirica. Una delle prime prove generali di un ingresso del tema icario nel capace alveo della casistica erotica lo offre la cosiddetta poesia cortigiana, nella persona del Tebaldeo:

Di gran periglio son le grandi imprese!

De ciò Phetonte et Icaro fan fede:

questo, perché a imitar gli ocei se diede, quello, perché del padre il carro ascese.

E a me del troppo ardir che 'l cor mio prese non men doglioso fin nato si vede: ché, se a l'antica auctorità si crede, il fulmine un, l'altro il sol caldo offese, me la fiamma de Amor che assai più incresce. E se l'un cade in mar, l'altro nel fiume, dentro a una acqua cadi io che ognhor più cresce. 
Né importar vòl quel carro e quelle piume altro se non che chi del suo grado esce convien che alfin in pianto si consume.

$$
(\text { Rime } 347)^{32}
$$

A parte il consueto gusto per le simmetrie - dagli esiti qui un poco legnosi-eper le allusioni argute, si registra già nel sonetto la trasposizione metaforica che si fisserà come canone: ilsole èilfuoco dellapassione amorosa (o lo sguardo dell'amata), ${ }^{33}$ ilmare le lacrime sorte dalla sofferenza a peril suo esito infelice, in una rilettura del mito che tende ad una forte drammatizzazione dei dati soggettivi, cosi come si trovano cristallizzati dalla convenzione lirica. Si noti, inoltre, che viene mantenuta l'esemplarità parallela Icaro-Fetonte, e che, ancor più di quanto non accada nell'esempio citato del Petrarca, la chius a del sonetto esplicita volutamente la quadratura gnomica della vicenda narrata; aspetti, questi, che conferiscono una certa patina arcaizzante al componimento. Quanto al sintagma troppo ardir, che conoscerà come vedremo lunga fortuna, ${ }^{34}$ esso sembra derivare da un'altra delle "prove di volo» petrarchesche: Rvf. I77. ${ }^{35}$ Un secondo sonetto icario del Tebaldeo, Ove ne vai, cor mio cieco? misura, ripete nella sostanza $i$ modi e le caratteristiche del componimento gemello, ma introducendo, in più, il tema dell'uccellino che brucia le sue ali approssimandosi troppo al fuoco. ${ }^{36}$

Di una sostanziale influenza tebaldeana ${ }^{37}$ occorre parlare per il sonetto ariostesco Del mio pensier, che così veggio audace, sorta di drammatizzazione di un'impari lotta tra un desiderio temerario e onnipotente e di una ragione neghittosa:

Del mio pensier, che così veggio audace, timor freddo com'angue il cor m'assale; di lino e cera egli s'ha fatto l'ale, disposte a liquefarsi ad ogni face.

E quelle, del desir fatto seguace, spiega per l'aria e temerario sale, duolmi ch'a ragion poco ne cale, che devria ostarli e sel comporta e tace.

Per gran vaghezza d'un celeste lume temo non poggi sì, ch'arrivi in loco dove s'incenda e torni senza piume.

Seranno, oimè! le mie lacrime poco per soccorrergli poi, quando né fiume né tutto il mar potrà smorzar quel foco.

Se la rima ale-sale potrebbe denunciare l'influenza del sonetto 600 del Tebaldeo, ritorna l'influenza di Rvf. I77 per la rima lume-piume-fiume delle 
terzine (di cui è conservata anche la medesima struttura: $C D C D C D$ ); ma, tra tanto igneo vigore, non manca anche un certo sentore di dantesco bruciato: la serie loco-poco-foco, infatti, connota alcuni luoghi classici della prima cantica, come il castello degli spiriti magni, l'episodio di Farinata e quello di Ulisse. $^{38}$

Prova non del tutto riuscita di elaborazione lirica del tema icario è quella del Bandello, ${ }^{39}$ impegnata in una ricostruzione narrativa della vicenda dagli esiti improbabili, data la brevitas del sonetto, dunque fatalmente debitrice al racconto ovidiano, ${ }^{40}$ chiude il tutto una terzina che traspone, con un certo schematismo, la vicenda su un piano erotico, invocando una ragione che tuttavia sottrae l'amante dal rischio di essere semplicemente schernito.

Una radicale svolta nell'elaborazione del motivo si ha col già citato sonetto LXXIX del Sannazaro, che recupera tutta la drammaticità del mito in chiave solenne, di storia collettiva e non più di vicenda privata, sulla base, come s'è detto, di precedenti classici: in particolare Seneca, di cui però viene rovesciato il giudizio morale, poiché si assegna allo sfortunato tentativo di Icaro la gloria di una fama immortale. Ecco dunque il sonetto:

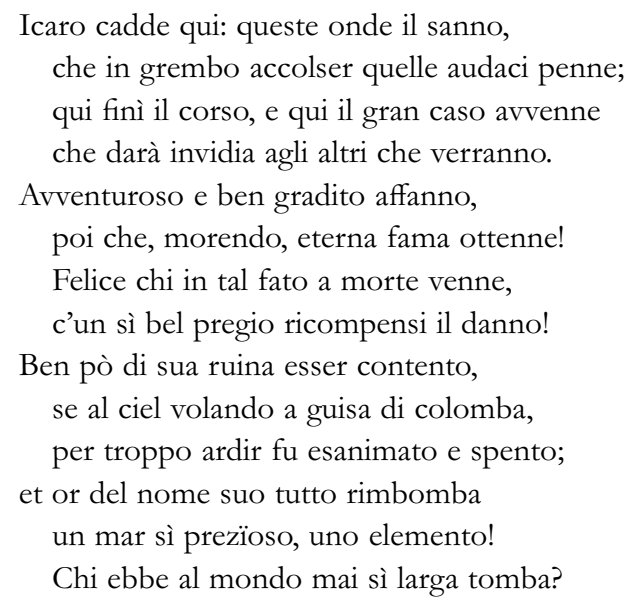

(Rime Lxxix)

Giova qui ripetere quanto ha osservato il Velli: il sonetto presenta una insolita (per Sannazaro) coincidenza di metro e sintassi, in una struttura rigidamente bipartita nelle quartine, inaugurata dal ritmo martellante dei deittici - all'effetto della labiovelare si assomma poi l'occlusiva velare sorda: Icaro cadde qui: queste ( $I$ ); accolser quelle (2); qui finì il corso, e qui il gran caso (3) - e resa più tesa e risentita dagli ossimori gran caso $e$ gradito affanno $e$ da un più generale gioco chiastico. Si può dire che questo perfetto "monumento da tavolo" si erga a compendio esemplare di tutta la 
tradizione icaria: vi si legge, tra le altre cose, una replica perentoria a Seneca (a male pensantur magna ruinis, Herc. Oet. 69, si oppone Ben po’ di sua ruina esser contento) e un allusione alla vicenda parallela di Fetonte (per troppo ardir riprende il magnis tamen excidit ausis di Ovidio, Met. II, 328) che, come s'è detto, rappresenta in un certo senso il completamento di quella icaria per la presenza di un epitaffio, assente nella prima. La sepoltura di Icaro, a differenza del racconto ovidiano, in Sannazaro non esiste se non come larga tomba, come spazio non delimitabile di una fama immensa: quel mare, ${ }^{4 \mathrm{I}}$ cioè, che inghiotti lo sfortunato eroe non facendone scomparire le tracce, ma consegnandole per sempre alla memoria; larga tomba che rovescia una volta ancora una serie di rilievi classici, poi sannazariani, centrati su un'angustia del sepolcro che fatalmente evidenzia tutta la fragilità e la pochezza della vita umana, ${ }^{42}$ vera ossessione di un poeta che trova $i$ suoi accenti più vibranti ogni volta che avverte un oggetto come per sempre perduto. Prova affine al sonetto LXXIX, per la presenza di un nome glorioso che non rimane confinato nel breve perimetro dell'urna, ma distende la sua presenza lungo tutta la vastità degli elementi è il sonetto Spargi di lauri, palme e mirti foglie (Disp. 2$).{ }^{43}$

Non poca eco alla fama alata di Icaro danno le nasali che invariabilmente si trovano in sede di rima, e che passano, in crescendo, da semplici doppie (alternanza ANNO-ENNE nelle quartine) ad un nesso consonantico che alterna nasale + sorda a nasale + sonora (anche qui in crescendo di sonorità: ENTO-OMBA-ENTO, OMBA-ENTO-OMBA: senza contare l'enjambement del $v$. II che ne prolunga l'effetto). E qui andrà ricordato, però, Dante:

$$
\begin{aligned}
& \text { E 'l duca disse a me: "Più non si desta } \\
& \text { di qua dal suon de l'angelica tromba, } \\
& \text { quando verrà la nimica podesta: } \\
& \text { ciascun rivederà la trista tomba, } \\
& \text { ripiglierà sua carne e sua figura, } \\
& \text { udirà quel ch' in etterno rimbomba". } \\
& \text { (Inf. 6,94-99) }
\end{aligned}
$$

Dante non sembra però fruito direttamente, ma attraverso Petrarca, Rvf. I87, in cui ritroviamo, oltre a tomba e rimbomba, anche il dantesco tromba $e$ il sannazariano colomba, nell'ambito di un contesto già orientato verso l'accordo fama-sepoltura:

Giunto Alexandro a la famosa tomba del fero Achille, sospirando disse: O fortunato, che sí chiara tromba trovasti, et chi di te sí alto scrisse! 
Ma questa pura et candida colomba, a cui non so s' al mondo mai par visse, nel mio stil frale assai poco rimbomba: cosí son le sue sorti a ciascun fisse.

$$
(R v f . \mathrm{I} 87, \mathrm{I}-8)^{44}
$$

Sequenza rimica che ritroviamo nella sua completezza nel sonetto $L V$ di Sannazaro, quasi a conferma della pertinenza del luogo petrarchesco. ${ }^{45} \mathrm{E}$, visto che si parla di colombe, non si può dimenticare il valore che tale riferimento assume nel già citato sonetto LXXXI del Canzoniere (in cui di nuovo abbiamo la rima rimbomba-colomba nei terzetti): un valore a cui il nostro sonetto in qualche modo sembra alludere. Sequela, questa, che appare piuttosto ricca di riprese in area meridionale. ${ }^{46}$

Eproprio in un'altropoeta meridionale, il Tansillo, a conferma di unaprimazia che l'area napoletana ${ }^{47}$ condivide con quella a adana nell' elaborazione lirica della tematica icaria, troviamo la ripresa piu interessante della lettura icaria del Sannazaro, articolata in una suite di ben tre sonetti (II, III e CII).

Amor m'impenna l'ale, e tanto in alto le spiega animoso mio pensiero, che, ad ora ad ora sormontando, spero a le porte del ciel far novo assalto.

Tem’io, qualor giù guardo, il vol tropp'alto, ond'ei mi grida e mi promette altero, che, s'al superbo vol cadendo, io pero, l'onor fia eterno, se mortal è il salto.

Ché s'altri, cui disio simil compunse diè nome eterno al mar col suo morire, ove l'ardite penne il disgiunse, ancor di me le genti potran dire: - Quest'aspirò a le stelle, e s'ei non giunse, la vita venne men, ma non l'ardire! -

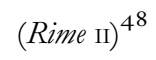

Poi che spiegate ho l'ale al bel disio, quanto per l'alte nubi altier lo scorgo, più le superbe penne al vento porgo, e, d'ardir colmo, verso il ciel l'invio.

Né del figliuol di Dedalo il fin rio fa ch'io paventi, anzi via più risorgo. ch'io cadrò morto a terra ben m'accorgo; ma qual vita s'agguaglia al morir mio? 
La voce del mio cor per l'aria sento:

- Ove mi porti, temerario? China, ché raro è senza duol troppo ardimento! -

Non temer (rispond'io) l'alta rovina; poiché tant'alto sei, mori contento, se 'l ciel sì illustre morte ne destina

(Rime III)

Tansillo sta al gioco del Sannazaro, riprendendo il tono eroico e sublime che il sonetto LXXIX aveva saputo tanto dottamente evocare, ${ }^{49}$ e rilancia. Alla transizione tra passato e presente eterno della fama in Sannazaro (cadde, accolser, ecc./ et or [...] rimbomba), comunque al servizio di un'immobilità statuaria e immodificabile, Tansillo oppone un presente in cui la figura dello speaker del testo e quella di Icaro coincidono, e dove la tensione dinamica viene rafforzata dal parallelo ricorso alla sermocinatio o al fulminante scambio dialogico. ${ }^{50}$ Il poeta recupera, in funzione drammatica, anche il timbro sentenzioso che costituiva una dei contrassegni primitivi del tema (si ricordino i già citati $\mathrm{Rvf}$. 307, 7: «[...] A cader va chi troppo sale» e Tebaldeo, 600 - estrav. -: "Non sciai che quanto più si monta e sale, / tanto è più la caduta aspera e dura?»): ${ }^{\mathrm{I}}$ "Che raro è senza duol troppo ardimentol» (III, $I I)$, «[...] ch'a chi troppo sale, / cadendo poi, tanto più noce il salto» (CII, 7-8). Col Tansillo, si può dire che il "canone icario" sia ormai fissato; le uniche novità potranno venire al più dalla contaminazione del mito col motivo della farfalla $^{52}$ (o dell'uccello) che brucia le sue ali attirata dalla fiamma, aspetto del resto già presente, come s'è visto, nel Tebaldeo, nel notevole sonetto di Bernardo Tasso Io pur m'inalzo con dedalee piume, ${ }^{53}$ e ancora nel Tansillo del madrigale S'un Icaro, un Fetonte, ${ }^{54}$ e recepito peraltro in modo diffuso dalla lirica spagnola e francese. ${ }^{5}$

E proprio in quest'ultimo ambito si vorrebbe, prima di prendere definitivamente congedo col Tasso, condurre una rapida e certo non esaustiva verifica della diffusione del motivo icario, quale esempio del decisivo influsso operato dai poeti italiani sui loro sodali d'oltralpe. Si può partire con un autore come Mellin de Saint Gelais (I49I-I558), poeta ufficiale di corte tra l'eclissi di Marot e l'ascesa di Ronsard, buon conoscitore della poesia italiana anche grazie ai suoi soggiorni a Bologna e Padova. Il sonetto che citiamo ci riporta al gia ricordato filone pseudo-lucianeo, essendo premesso all'operetta Advertissement sur les jugemens d'astrologie (Lione, J. de Tounes, I546):

Ne craignez point, plume bien fortunée, qui vers le ciel vous allez eslevant, faire ruine Icarus esuyvant, qui trop haussa l'aile mal empennée: 
du beau soleil ou estes destinée vous n'irez point la chaleur esprouvant; mais deviendrez, sous ses rays escrivant, de sa clarté belle et enlumininée.

Et si, volant parmi le grand espace, de ses vertus quelque feu concevez, ja moins pourtant ne vous en eslevez.

Ce ne sera feu qui brusle ou desface mais bien fera sa divine estincelle, comme Phoenix revivre vous et elle. ${ }^{56}$

L'esempio icario questa volta è presentato come eventualità irrealizzata, pericolo da cui si è preservati grazie ad una clarté non ustoria, anzi capace di trasmettere le sue benefiche virtù, secondo un canovaccio neoplatonico ben collaudato. Un possibile raccordo intertestuale, cronologia permettendo, ${ }^{57}$ può essere stabilito col sonetto III del Tansillo, v. I2: "Non temer", rispond'io, "l'alta rovina"》 (Ne craignez point [...] / faire ruine), e direi che dal senso generale di quel componimento, l'eroica sfida alle altezze che si conclude, a differenza di quanto era successo ad Icaro, in modo vittorioso e quasi con una rinascita ("Né del figlinol di Dedalo il fin rio / fa ch'io paventi, anzi via più risorgo»), è tratta l'immagine finale della fenice. Sta di fatto che qui non appare tanto in questione l'astrologia, quanto l'amore per una donna, il beau soleil fonte di tutte le virtù a cui il sonetto allude: può dunque darsi che esso sia stato composto in precedenza e poi, secondo una prassi comune nel Cinquecento, aggiunto come premessa all'Advertissement.

Un autore che percorre in lungo e in largo il topos icario, esplorando tutte le valenze offerte dalla tradizione, è senza dubbio Du Bellay. Nell'Olive, sull'abbrivio di Orazio, Carm. IV, II, ${ }^{58}$ il poeta canta l'elogio del cigne Ronsard, del suo vol audacieux; ma ecco fare improvvisamente la sua comparsa, repentino spettro di una possibilità priva di sèguito, appunto la caduta di Icaro, come mostra l'importante spia sannazariana (LXXIX, I4) del large tombeau:

Quel cigne encor' des cignes le plus beau te prêta l'aele? et quel vent jusqu'aux cieulx te balança le vol audacieux, sans que la mer te fust large tombeau? ${ }^{59}$

Decisamente orientata in altro senso una seconda occorrenza del tema, tutta orientata verso la connotazione petrarchesca del volo come desiderio:

Mais (ô moy sot!) de quoy me doy-je plaindre, fors du desir, qui par trop hault ataindre, me porte au lieu, où il brusle ses aesles? 
puis moy tumbé, Amour, qui ne permet finir mon dueil, soudain les luy remet, renouvelant mes cheutes eternelles.

(Olive xxxviI, 9-14)

In questo caso vale forse la pena risalire fino a Rvf. 23 (stanza della trasformazione in cigno): «allor che folminato et morto giacque / il mio sperar che tropp'alto montava), con la sua allusione al mito di Fetonte (ma si ricordi il vol tropp'alto di Tansillo II, 5). Nell'Olive non manca, infine, nemmeno la variante più rarefatta del motivo, quella che, rifacendosi alla lettura neoplatonica, vede nel volo l'anelito dell'anima a ritornare nel regno di una bellezza incorruttibile:

Que songes-tu mon ame emprisonnée?

Pourquoy te plaist l'obscur de nostre jour,

Si pour voler en un plus cler sejour,

Tu as au dos l'aele bien empanée?

(Olive cxiII, 5-8)

Anche Ronsard ricorre al motivo icario con finalità metatestuali (per discutere, cioè, di poeti e poesia), ed è ancora la cifra oraziana-ovidianasannazariana del nom che si impone nelle Odes, sia pure in chiave ironica. ${ }^{60}$ In effetti egli sembra particolarmente refrattario alla lettura eroico-spirituale del mito, come mostra perentoriamente Sonets pour Hélène XLII, I-8:

En choisissant l'esprit vous estes mal-apprise, qui refusez le corps, à mon gré le meilleur: de l'un en l'esprouvant on cognoist la valeur, l'autre n'est rien que vent, que songe et que feintise.

Vous aimez l'intellect, et moins je vous en prise: vous volez, comme Icare, en l'air d'un beau malheur: vous aimez les tableaux qui n'ont point de coleur. Aimer l'esprit, Madame, est aimer la sottise. ${ }^{61}$

Diverso è l'atteggiamento del poeta quando il tema appare riferito a un contesto morale ${ }^{62}$ e, soprattutto, erotico: è il caso della versione del citato sonetto ariostesco Del mio pensier, che così veggio audace ${ }^{\sigma_{3}}$ e della più notevole prova icaria di Ronsard, il componimento di apertura dei Sonets et madrigals pour Astrée:

Dois-je voler emplumé d'esperance, ou si je dois, forcé du desespoir, 
du haut du Ciel en terre laisser choir mon jeune amour avorté de naissance?

Non, j'aime mieux, leger d'outrecuidance, tomber d'enhaut, et fol me decevoir, que voler bas, deussé-je recevoir pour mon tombeau toute une large France.

Icare fit de sa cheute nommer, pour trop oser les ondes de la mer: et moy je veux honorer ma contree de mon sepulchre et dessus engraver, Ronsard voulant aux astres s'eslever, fut foudroyé par une belle Astrée.

Qui il codice eroico di Sannazaro e Tansillo è preso decisamente in carico: per quanto riguarda il primo, large France (8) richiama indubitabilmente la larga tomba di LXXIX I4, pour trop oser $e$ les ondes de la mer (Io) il sintagma per troppo ardir e le onde testimoni della caduta ( $L X X I X$ II $e$ I); per Tansillo, emplumées d'espérance (I) rimanda a II I e 3-4: Amor m'impenna l'ale $e$ spero / [...] far novo assalto. Anche la struttura d'insieme risponde ad una logica rigorosa e perentoria, dividendosi in una prima quartina retoricamente interrogativa centrata sulle istanze enunciative dell'io, una seconda di inappellabile replica, e stabilendo per via esplicita, nelle terine, un parallelo tra la vicenda icaria e quella del poeta. Tuttavia, nonostante il sonetto si concluda a modo d'epigrafe, la chiusa non appare per nulla solenne, ma concettosamente galante, quasi in anticlimax rispetto al resto del componimento, pungente e un poco contradittoria ${ }^{64}$ coda epigrammatica che conferma la vocazione anti-aulica dell'autore.

L'ultimo autore francese di cui vorrei occuparmi è Philippe Desportes, $i$ cui rapporti con la tradirione poetica italiana sono stati oggetto di una nutrita serie di saggi. ${ }^{65}$ Entro subito in medias res, accantonando riferimenti meno significativi, ${ }^{66}$ e affrontando immediatamente il testo d'apertura de Les amours d'Hippolyte:

Icare est cheut icy le jeune audacieux, ${ }^{67}$ qui pour voler au Ciel eut assez de courage: icy tomba son corps degarni de plumage, laissant tous braves coeurs de sa cheutte envieux.

o bien-heureux travail d'un esprit glorieux, qui tire un si grand gain d'un si petit dommage! o bien-heureux malheur plein de tant d'avantage, qu'il rende le vaincu des ans victorieux! un chemin si nouveau n'estonna sa jeunesse, 
le pouvoir luy faillit mais non la hardiesse,

il eut pour le bruler des astres le plus beau.

il mourut poursuivant une haute adventure,

le ciel fut son desir, la Mer sa sepulture

est-il plus beau dessein, ou plus riche tombeau?

Occorre riflettere, sia detto tra parentesi, sulla natura programmatica che il tema icario sembra assumere nella lirica francese nella seconda metà del Cinquecento, conquistando, come accade per $i$ Sonets et madrigals pour Astrée di Ronsard (I578) e per Les amours d'Hippolyte (I573) di Desportes, una posizione incipitaria che va intesa, a mio giudizio, come adibizione metatestuale di un manifesto di poetica: è una caratteristica, come si è avuto modo di sottolineare, presente in nuce anche nella tradizione classica. Tornando allo specifico del sonetto, non sfuggirà la sua stretta adesione al testo sannazariano, ${ }^{68}$ evidente sin dal tentativo dell'incipit di riprodurne gli effetti metrici e fonetici. Desportes sembra impegnato soprattutto in uno sforzo di semplificazione del suo modello; sopprime innanzitutto il riferimento all'asse di osservazione della vicenda icaria, il mare (queste onde [...] / che in grembo accolser), venendo cosi a compromettere però la simmetria del sonetto LXXIX, che appunto in ambito marino trova la sua conclusione. Sul piano lessicale il confronto è senz'altro in perdita: grand gain (G) suona meno sublime di bel pregio, cosi come petit dommage tende a svilire il danno (8). Forse l'aspetto più interessante dell'adattamento di Desportes è la compattezza strutturale, ${ }^{69}$ ottenuta attraverso una particolare ricerca di simmetria (ad esempio nei membri $\mathrm{O}$ Bien heureux travail [...] qui tire / O Bien heureux malheur [...] qu'il rende), e gioco ossimorico: heureux travail, gran gain, petit dommage, bien-heureux malheur, vaincu, victorieux, ciel, sepulture. Non a caso la sezione del componimento del Sannazaro rimaneggiata in modo più radicale è la prima terzina: al suo andamento argomentativo un poco lambiccato e sinuoso (Ben po' [...] esser / se al ciel volando / per troppo ardir fu) vengono sostituiti tre lapidari membri sintatticamente autonomi, tutti di qualificazione positiva (la novità del chemin ${ }^{70}$ e la jeunesse, l'audacia, infine la somma nobiltà dell' astro che causa l'epilogo della vicenda), proprio quello mediano (le pouvoir luy faillit mais non la hardiesse) denuncia la presenza per il sonetto di Desportes di una lettura contaminata, poiché si richiama a Tansillo II I4 (La vita venne men, non già l'ardire). ${ }^{7 \mathrm{I}}$ Siamo, insomma, su una linea diametralmente opposta a quella ronsardiana, una linea che, nell'esaltare il coraggio dell'impresa icaria, la sua oltranza, tende a privilegiare quello che Weise ba chiamato l'«ideale eroico del Rinascimento». ${ }^{72}$

L'epilogo di questa lunga parabola spetta dunque, come si diceva, al Tasso, 
poeta destinato a trovarsi sempre sul crinale di differenti e talvolta contradditorie situazioni, nella cui opera da un lato trovano compendio le complesse risultanze della tradizione; dall'altro, per la prima volta, emergono all'interno della lettura del mito inedite implicazioni autobiografiche, ${ }^{73}$ già presaghe di più moderne riflessioni sulla dolorosa, inevitabile frattura tra io poetico $e$ mondo. Prosegue nel Tasso il lavoro di contaminazione a livello tematico, che già Tebaldeo e Tansillo avevano avviato, richiamando in sede icaria il topos dell'uccellino e della farfalla: il Tasso sviluppa in particolare due sintesi care alla tradizione: quella con il mito di Fetonte, ${ }^{74}$ che anzi appare la figura piu in rilievo, e quella, oraziana, relativa al cigno. Ignorando taluni sviluppi secondari del motivo, ${ }^{75}$ possiamo senz'altro citare il componimento in cui il mito acquisisce la sua piena centralità, il sonetto 794 delle Rime:

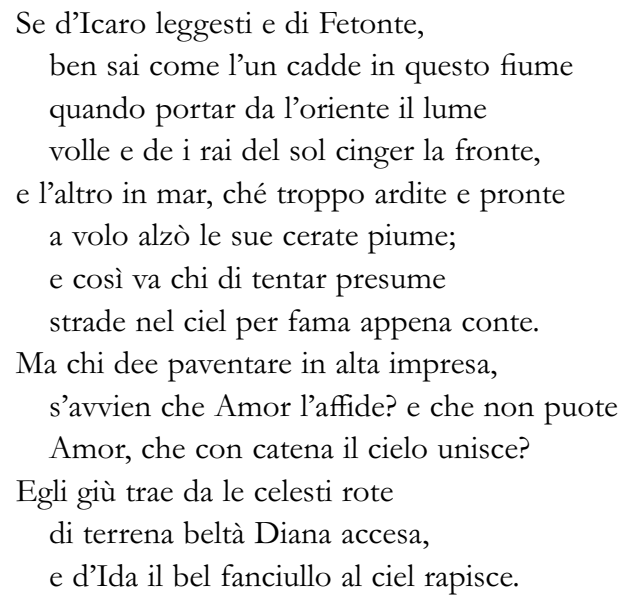

I contrassegni della tradizione icaria sono ben visibili: troppo ardite $(5)^{76}$ ripete il sannazariano troppo ardir (LXXIX, II) e il tansilliano troppo ardimento (III, II), e cosi dicasi per il tono didascalico dei vv. 7-8, che abbiamo visto essere una costante dell'elaborazione lirica del mito. Ma è nelle terzine che il Tasso gioca le sue risorse di poeta doctus, ignorando la possibilità di una scontata chiusa erotico-galante, e recuperando in tutta la sua ricchezza una chiave neoplatonica di lettura che interpreta la catena del v. II come universale "circolazione amorosa" capace di unire Dio alle creature e viceversa, secondo l'esegesi di Iliade VIII, I8-27 esposta nel dialogo Il Messaggiero; ${ }^{77}$ a confermare tale circolarità, l'ultima terzina accosta ad una discesa del divino nell'umano (Endimione) una salita dell'umano al divino (Ganimede).

A conferma dell'originalità degli esiti del tema icario nella lirica tassiana possiamo leggere un nuovo sonetto, il 747, indirizzato al Duca di Mantova e 
composto durante la reclusione in Sant'Anna, recante le stigmate di una disperazione a cui presta accenti persino il Salmo 130, De profundis clamavi ad te, Domine:

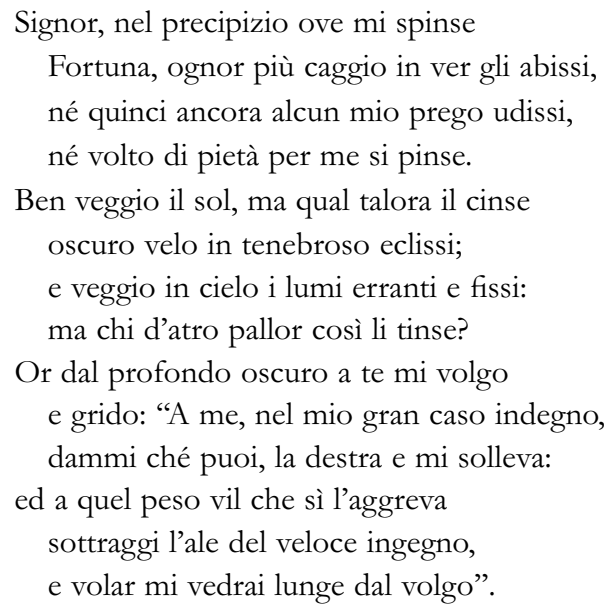

Il sintagma sannazariano gran caso (LXXIX 3) chiarisce subito di quali ale e volo si stia parlando: ma qui la bruciante materia autobiografica agisce come ipotesi innovativa, contribuendo a creare all'interno del del mito, per cosi dire, una mitologia del personaggio: il volo non è espressione di semplice desiderio, non di ardimento e volontà di sfida intellettuale, non è fuga neoplatonica dal carcere del corpo o della materia, ma liberazione da un'oppressione vissuta in prima persona, riscatto da una caduta storicamente registrabile, seppur reinventata alla luce della tradizione letteraria. Non sarà senza significato che, nella Liberata, il gran caso sia attribuito a Lucifero e ad Ulisse, ${ }^{78}$ segno evidente del significato sublime ed estremo che il Tasso attribuisce alla formula. S'è detto dello stretto legame tra le figure di Icaro e Fetonte nell'opera tassiana: nel sonetto 738 a Luigi d'Este, tutto incentrato sul secondo mito, il gran caso diviene, a connotare ancora una volta la vicenda personale del poeta, aspro $e$ maligno caso, confortato solo dalla finale speranza di un'oraziana metamorfosi in cigno, la sola che potrebbe consentire un volo liberatorio. ${ }^{79}$ "I poeti sono cosa volatile, come dice Socrate ne l'Ione», chioserà Tasso nel commento alle proprie Rime: ${ }^{\circ \circ}$ ma l'esperienza cortigiana insegna che essipossono facilmente essere abbattuti; per questo, forse, egli sceglierà per sé l'epiteto quasi baudelairiano di "cigno infelice».8 I Saranno gli stessi contemporanei, finalmente, a riconoscere nell'emblema icario della caduta $^{82}$ e in quello del cigno prigioniero ${ }^{83}$ la parabola esemplare che farà del Tasso l'alfiere di una tragica modernità. 
* Il presente contributo costituisce l'approdo di un percorso di lunga data, che ha avuto come tappe intermedie di elaborazione due conferenze: la prima, tenuta a Parigi all'École des Hautes Études en Sciences Sociales nel 1998 (dal titolo La cbute d'Icare comme modéle de metamorphose stilistique et thematique dans la poèsie italienne et française du XVI siècle - et dans la peinture de Brueghel l'Ancien -); la seconda alla Faculté des Lettres di Ginevra nel 2003 (Envol et chute d'Icar: modèles classiques et textes poétiques italiens et françaises du XVI siècle).

I. G. Bachelard, L'air et les songes. Essai sur l'imagination du mowvement, Paris, Corti I943, p. I I I.

2. Che tuttavia ritorna come riferimento cursorio anche nei Tristia e nei Fasti. Nei Tristia l'amara esperienza di esiliato suggerisce a Ovidio di interpretare senza filtri l'insegnamento morale del racconto icario, non senza che faccia nuovamente capolino, con chiaro significato simbolico, la metafora nautica, a rappresentare un percorso biografico assai accidentato: cfr. Ovidio, Tristia I, I, 89-92 «Et mea cumba, semel vasta percussa procella, / illum, quo laesa est, horret adire locum. / Ergo cave, liber, et timida circumspice mente, / ut satis a media sit tibi plebe legi! / dum petit infirmis nimium sublimia pennis / Icarus, aequoreis nomina fecit aquis». Si veda anche III, 4, I 5-25: «Dum mecum vixi, dum me levis aura ferebat, / haec mea per placidas cumba cucurrit aquas. / Qui cadit in plano - vix hoc tamen evenit ipsum -, / sic cadit ut tacta surgere possit humo; / at miser Elpenor tecto delapsus $\mathrm{ab}$ alto / occurrit regi debilis umbra suo. / Qui fuit, ut tutas agitaret Daedalus alas, / Icarus immensas nomine signet aquas? / Nempe quod hic alte, demissius ille volabat; / nam pennas ambo non habuere suas». Cfr. infine Ovidio, Fasti IV, 283-84.

3. Cfr. Ovidio, Ars amandi II 2 1: «Hospitis effugio praestruxerat omnia Minos».

4. Ritroviamo il tema del cigno in relazione con quello icario in una seconda ode, la IV, II, dedicata a Pindaro, il «Dircaeum [...] cycnum» (v. 25) il cui volo appare inimitabile, pena una rovinosa caduca quale quella di Icaro (cfr. vv. I-4).

5. Cfr. il volume di M. Jacob, Schwanengefabr: das lyrische Icheichen des Schwans, München, Carl Hanser Verlag, 2000, che dedica appunto un capitolo all'ode II, xx di Orazio.

6. Cfr. Virgilio, Aen. III 304 «tumulum [...] inanem» e Ovidio, Met. VI, 568 «inane sepulcrum».

7. In questo contesto non tanto la velocità, quanto la solennità conta; si tenga poi presente che «Daedaleo ocion» creerebbe iato, compromettendo la regolarità metrica (cfr., tra le molte esegesi, R. G. M. Nisbet - M. Hubbard, A Commentary on Horace: Odes. Book II, Oxford, Clarendon Press, 1978, p. 344).

8. Non si accorda con questo contesto la congettura di Bentley che, sulla base di una supposta contraddizione tra la caduta e l'ottenimento della fama da parte di Icaro, emenda notior in tutior.

9. G. Velli, Un sonetto del Sannazaro in Tra letteratura e creazione. Sannazaro - Alfieri - Foscolo, Antenore, Padova I983, pp. 57-72.

Io. Precisamente Ovidio, Met. I, $750-79$; II, I-367 e VIII I 83-235.

II. Ovidio, Ars am. II 21-96.

I 2. Ovidio, Tristia I, I, 79-90 e III, 4, 17-30.

I 3. La formula attraverso cui si realizza la condanna dell'abbandono dell'aurea mediocritas è quella del medium iter, che ricorre sia in Herc. Oet. 675 e 683, sia in Oed. 899-900. Tuttavia si veda anche ciò che Seneca afferma in De vita beata 20, 2, parlando della difficoltà per il 
saggio di realizzare le proprie deliberazioni: «Quid mirum, si non escendunt in altum ardua aggressi? Sed si vir es, suspice, etiam si decidunt, magna conantis». Si noti che a 20, s è posta a suggello dell'argomentazione proprio la citazione ovidiana di Met. II, 328 relativa alla tomba di Fetonte.

I4. Va registrato tuttavia un «damnosas erudit artes» di Met. VIII, 2 I 5 . Le «audaci penne» del v. 2 del sonetto sanazzariano sembrano rinviare alle «audaces alas» di Heroid. XVII, 49.

I 5. Cfr. Ovidio, Met. VIII, 229-30: «oraque caerulea patrium clamantia nomen / excipiuntur aqua, quae nomen traxit ab illo»; Ars am. I, 96: «Ossa tetigit tellus, aequora nomen habent»; Trist. I, I, 90: «aequoreis nomina fecit aquis»; Trist. III, 4, 22: «Icarus inmensas nomine signet aquas»; infine Fast. IV, 284 «et vastae nomina fecit aquae»: il tutto sulla base di OrazIO, Carm. IV, 2, 3-4: «vitreo daturus / nomina ponto», ancora riferito al mito icario. Per inciso, proprio gli ultimi due riferimenti ovidiani forniscono lo sfondo intertestuale più congruo ai VV. I 2-I 3 del sonetto LXxix di Sannazaro: «et or del nome suo tutto rimbomba / un mar sì spazioso, uno elemento».

I6. Giovenale, Sat. III, 74-80: «[...] Ede quid illum / esse putes. quemuis hominem secum attulit ad nos: / grammaticus, rhetor, geometres, pictor, aliptes, / augur, schoenobates, medicus, magus, omnia nouit / Graeculus esuriens: in caelum, iusseris, ibit. / In summa non Maurus erat neque Sarmata nec Thrax / qui sumpsit pinnas, mediis sed natus Athenis».

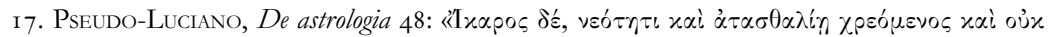

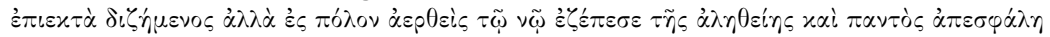

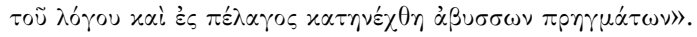

I 8. Cito dall'ed. a cura di E. Garin, Firenze, Vallecchi, I946-52.

i 9. Andrea Alciati, Emblemata [i 53 I], che cito dall'ed. Venezia, Aldo, i 546.

20. Pontus de Tyard, Mantice. Discours de la verité de divination par astrologie, ed. critica a cura S. Bokdam, Genève, Droz, I990, p. I02; cfr. L. Sozzi, "Coeli cupidine tractus": note sul mito di Icaro nella poesia del Rinascimento, in Mélanges de poétique et d'histoire littéraire du XVI siècle offerts à Louis Terreaux, a cura di J. Balsamo, Paris, Champion, I994, pp. 175-203, in particolare pp. I77-78.

2 I. Cfr. N. Rudd, Dedalus and Icarus from Rome to the end of the Middle Ages, in AA.vv., Ovid Renewed. Ovidian Influences on Literature and Art from the Middle Ages to the Twentieth Century, a cura di Ch. Martindale, Cambridge, Cambridge Unv. Press, I988, p. 28.

22. Giovanni di Salisbury, Polycraticus, VIII, xxiII (PL CXCIX, 809): «Phaeton in fabulis, dum paternos currus affectat, incendit orbem, et tandem miseratione dei, et ipse succensus corruit praeceps, curru disjecto. Tunc: Isse diem sine sole ferunt: [OvIDIO, Trist. V, VIII, 3 I] et dum flagrat Ecclesia succensa schismate, Christus videtur abesse. Icarus quoque, dum elatus juvenili levitate fertur in coelum, marinis fluctibus mergitur. Dejectus enim est dum allevaretur. Subvectio siquidem impiorum, gravioris ruinae praeparatio est».

23. Cfr. Ovidio, Met. VIII, 232: “"Icare”, dixit "ubi es? qua te regione requiram?”»; Ars am. II, 94: " "Icare”, clamat "ubi es, quoque sub axe volas?"».

24. Giovanni Boccaccio, Filocolo II, 67: «Ma certo la paura del misero Icaro, volante più alto che il mezzo termine posto dal maestro padre, non fu tale quando sentì la scaldata cera lasciare le commesse a penne, quale fu quella di Biancifiore, quando il grande grido si levò: - Ecco il siniscalco! -. Ella non morì, e non rimase viva». Come si vede, l'influenza dantesca si trasmette anche all'ultima battuta citata (cfr. Inf. 24, 25). 
25. Giovanni Boccaccio, Comedia delle ninfe fiorentine, L: «La saetta, dal mio arco mossa, tocca li segni cercati con volante foga; e le bianche colombe, pasciute negli ampi campi, gratulanti ricercan le torri; e gli stanchi cavalli, compiuto il corso, domandan riposo; e così l'opera mia, guidata per gli umili piani, temente d'Icaro li miseri casi, alla sua fine presente», Elegia di Madonna Fiammetta, VII, I: «Ma mentre che in questa disposizione mi tenevano dispettosa l'iddii, la Fortuna ingannevole, la quale alcuna volta per afliggere con maggiore doglia li miseri loro nel mezzo delle avversità, quasi mutata, si mostra con lieto viso, acciò che essi più abandonandosi a lei caggiano maggiore istoscio cessando la sua letizia (li quali sì come folli s'appoggiano allora ad essa, cotali abattuti si truovano, quale il misero Icaro nel mezzo camino, presa troppa fidanza nelle sue ali, salito a l'alte cose, da quelle nelle acque cadde, del suo nome ancora segnate); questa, me sentendo di quelli, non contenta de' dati mali, apparecchiandomi peggio, con falsa letizia indietro trasse le cose avverse e 'l suo corruccio, acciò che, più movendosi di lontano, non altramenti che facciano i montoni africani per dare maggiore percossa, più m'offendesse; $\mathrm{e}$ in questa maniera con vana allegrezza alquanto diede sosta alle mie doglie».

26. Francesco Petrarca, $R v f$. 177 , I-4: «Mille piagge in un giorno et mille rivi / mostrato m’à per la famosa Ardenna / Amor, ch'a' suoi le piante e i cori impenna / per fargli al terzo ciel volando ir vivi».

27. Petrarca, $R v f$. i 82, I 2-I 4: «[...] ché 'l mio bel foco è tale / ch'ogni uom pareggia; et del suo lume in cima / chi volar pensa, indarno spiega l'ale».

28. Più in generale, la tradizione l'aveva attribuita all'ingegno ed alla speculazione: si ricordi Boezio, De consolatione Philosophiae IV, I, I-5: «Sunt etenim pennae volucres mihi, / quae celsa conscendant poli; / quas sibi cum velox mens induit, / terras perosa despicit, / aeris immensi superat globum».

29. Come ha mostrato Santagata nel suo commento (Milano, Mondadori, I996, pp. 4I7-1 8) nell'espressione lievimi da terra si registra una chiara influenza di VIRGILIO, Georg. III, 8-9 «temptanda via est, qua me quoque possim / tollere humo victorque virum volitare per ora». Sempre Santagata affianca a Rvf. 8 I l'importante passo di Epyst. I, i 4: «quis dabit ut pennas posita gravitate columbae / induar alta petens, et post tot dura quiescam?», oltre a $R v f$. 264, 6-8: «mille fiate ho chieste a Dio quell'ale / co le quai del mortale / carcer nostro intelletto al ciel si leva», Rvf. 359, 39: "Quant'era meglio alzar da terra l'ali»; Rvf. 365, 3: «senza levarmi a volo, abbiend'io l’ale».

30. Naturalmente l'interpretazione neoplatonica del mito di Icaro ha origini molto più antiche: lo testimonia lo studio degli apparati funerari romani, su cui si veda S. Calderone, Il mito di Dedalo-Icaro nel simbolismo funerario romano, in AA.vv., Romanitas-Christianitas. Untersucbungen zur Geschichte und Literatur der römischen Kaiserzeit. Jobannes Straub zum 70. Geburstag am I8. Oktober Ig82 gewidmet, a cura di G. Wirth, K.-H. Schwarte, J. Heinrichs, Berlin-New York, W. de Guyter, 1982, pp. 749-67, che si fonda in parte sulle osservazioni di P. Courcelle, Quelque symboles funéraires du néo-platonisme latin. Le vol de Dédale - Ulysses et les sirènes, «Revue des études anciennes», 46 (1944), pp. 6 5-93. Courcelle ricorda come AмBrogio, De virginitate XVIII (PL XVI, 296) non esiti ad impiegare il sintagma remigium alarum, che Virgilio impiega nella descrizione dell'episodio di Icaro (Aen. VI, I9) per commentare il Salmo LVI, 2, «Et in umbra alarum tuarum sperabo». Petrarca riprende la medesima espressione in un contesto decisamente escatologico: Epystole sine nomine, XIX, I-I4 (cito dall'ed. a cura di U. Dotti, Roma-Bari, Laterza, 1974, p. 2 I 8): «Evasisti, erupisti, enatasti, evolasti. Bene est. Timebam, fateor, tibi, timebam anime tue ne post descensum ad inferos non cum vellet emergeret. Sciebam et Averni descensum facilem et apertum laberinthi limen, laboriosum atque operosum exitum. Dicebam mecum: "O si meus Alcides, o si Theseus meus ab inferis redeat! $\mathrm{O}$ ne mali pondus illum opprimat neque pedes ad virtutem volucres adamantine scelerum catene vinciant detineantque!' Inter hec vota hosque inter metus emersisti, 
Deo gratias, qui etiam ex inferno in se sperantes animas educit. Exoptatus, evocatus, expectatus advenis. His igitur primum terris redditus, que te illi Herebo crediderant, non Phebo ut Dedalus, sed Cristo alarum remigium consecrabis et cavebis ne unquam amplius Gnoson spectes».

31. Cfr. Giovanni Pico, Oratio de hominis dignitate, 25: «Scribunt interpretes Chaldaei verbum fuisse Zoroastris alatam esse animam, cumque alae exciderent ferri illam praeceps in corpus, tum illis subcrescentibus ad superos revolare» (cito dall'ed. a cura di E. Garin, in La cultura filosofica del Rinascimento italiano, Firenze, Sansoni, I 979, p. 238). Vedi anche, seppure in una accezione meno diretta, Marsilio Ficino, El libro dell'amore, Orazione 4, cap. IV (cito dall'ed. a cura di S. Piccoli, Firenze, Olschki, I987, p. 65): «L'anima subito da Dio creata, per uno certo naturale instincto in Dio suo padre si converte, non altrimenti che il fuoco, per forza de' superiori generato in terra, subito per impeto di natura a' superiori luoghi si dirizza; sì che l'anima inverso Iddio rivolta, da' razzi di Dio è illustrata. Ma questo primo splendore quando si riceve nella substanza dell'anima, che era per sé sanza forma, doventa scuro, e tirato alla capacità dell'anima, doventa proprio a'llei e naturale; e però per esso, quasi come a'llei equale, vede sé medesima e le cose che sono sotto lei, cioè e corpi, ma le cose che sono sopra lei per esso non vede. Ma l'anima per questa prima scintilla diventata già propinqua a Dio, riceve oltr'a questo un altro più chiaro lume pel quale le cose di sopra conosca. Ha adunque due lumi, uno naturale l'altro sopra naturale, pe' quali insieme congiunti, come con due alie, possa per la regione sublime volare».

32. Antonio Tebaldeo, Rime, a cura di J. J. Marchand e T. Basile, Modena, F. Cosimo Panini, I 992 .

33. Così,ad esempio, in Alfonso D’Avalos: «Alzar mi sento a volo in parte, ch'io / temo di non aver egual le penne / a quelle di colui che non ottenne / a l'alta impresa il fin del bel desio. // E nasce in me timor de l'ardor mio: / ch'a maggior volo par ch'ogn'ora impenne / l'ali amorose, e gir tant'alto accenne, / che tener mi convien quel ch'io desio. // Che se pur giungo al fin del mio viaggio, / qual fia quella difesa che mi scampi / dai raggi di duo soli ardenti e chiari? // Accese lui d'un sole il caldo raggio: / che fia di me, s'al folgorare, ai lampi / giungo vicin dei lumi alteri e chiari?» (in Libro quinto delle rime di diversi illustri signori napoletani ed altri nobilissimi ingegni, Venezia, Giolito, I 555, p. Io). Il dettato, conformemente alla propria altezza cronologica (l'Avalos nasce nel i 5 IO), risente in modo palese della riforma bembesca, con un più attento filtraggio di tessere e situazioni petrarchesche; si veda per «alta impresa» $R v f$. 7 I, 2 (più altre tre occorrenze contestualmente meno significative); per «bel desio» $R v f$. 25 , г о е 34, I; per «impenne» Rvf. I 77, 3; per «mi scampi» Rvf. 35, 5; quanto infine allo splendore dei «duo soli», cfr. Rvf. 3 I I, iо e Rvf. 352, 2.

34. Si ritrova infatti nel sonetto Lxxix del Sannazaro e nel madrigale S'un Icaro, un Fetonte del Tansillo.

35. Petrarca, Rvf. I77: «Mille piagge in un giorno et mille rivi / mostrato m’à per la famosa Ardenna / Amor, ch'a' suoi le piante e i cori impenna / per fargli al terzo ciel volando ir vivi. // Dolce m'è sol senz'arme esser stato ivi, / dove armato fier Marte, et non acenna, / quasi senza governo et senza antenna / legno in mar, pien di penser' gravi et schivi. // Pur giunto al fin de la giornata oscura, / rimembrando ond'io vegno, et con quai piume, / sento di troppo ardir nascer paura. // Ma 'l bel paese e 'l dilectoso fiume / con serena accoglienza rassecura / il cor già vòlto ov'abita il suo lume».

36. Tebaldeo, Rime, 600 (estrav.): «Ove ne vai, cor mio cieco? Misura / il corso tuo, racogli le sparse ale! / Non sciai che quanto più si monta e sale, / tanto è più la caduta aspera $\mathrm{e}$ dura? // Dovria Phetonte pur farte paura / et Icar, che 'l volare intese male. / Non è, se guardi a te, l'impresa equale: / non conviense a un mortal tanta ventura. // Siocco non voler far sì come quello / ucel che, incauto, simplicetto e stolto, / al lume corre, onde se 
brusa in ello. // Se un bel riso te invita, o un lieto volto, / fugilo, come fugge il lupo agnello, / ché spesso tra bei fiori è il serpe involto».

37. Cfr. R. Fedi, Petrarchismo prebembesco in alcuni testi lirici dell'Ariosto, in AA.vv., Ludovico Ariosto: lingua, stile e tradizione, Atti del Congresso di Reggio Emilia e Ferrara, a cura di C. Segre, Milano, Feltrinelli, I 976, pp. 283-302; ora (col titolo Preistoria di un canzoniere: le Rime di Ludovico Ariosto) in La memoria della poesia. Canzonieri, lirici e libri di rime nel Rinascimento, Roma, Salerno, 1990, pp. 83-1 I 5 .

38. Dante Alighieri, Inf. 4, 67-72: «Non era lunga ancor la nostra via / di qua dal sonno, quand'io vidi un foco / ch'emisperio di tenebre vincia. / Di lungi n'eravamo ancora un poco, / ma non sì ch'io non discernessi in parte / ch'orrevol gente possedea quel loco», Inf. IO, I9-24: «E io: "Buon duca, non tegno riposto / a te mio cuor se non per dicer poco, / e tu m'hai non pur mo a ciò disposto". / "O Tosco che per la città del foco / vivo ten vai così parlando onesto, / piacciati di restare in questo loco"»; Inf. 26, 76-84: «Poi che la fiamma fu venuta quivi / dove parve al mio duca tempo e loco, / in questa forma lui parlare audivi: / "O voi che siete due dentro ad un foco, / s'io meritai di voi mentre ch'io vissi, / s'io meritai di voi assai o poco / quando nel mondo li alti versi scrissi, / non vi movete; ma l'un di voi dica / dove, per lui, perduto a morir gissi"». Ma la serie ricorre anche tre volte nel Purgatorio (9, 25-30; 25, I16-20; 33, 4-9) e ben cinque volte nel Paradiso (7, I 2 I-26; I 5, I 3-18; I8, 103-108; 19, I30-35; 25, II 8-23).

39. Matteo Bandello: «Tocco dal fuoco d' i celesti rai / Icaro cadde in mar, ché 'l grand'ardire / tant'alto il fe' poggiar che più seguire / l'orme del Padre egli non seppe mai. // Dedalo pur dicea: - figliuol che fai? / Ove ne voli? Ahimè, frena il salire, / piega più basso l'ali; il tuo fallire / veggio che già m'apporta eterni guai -. // Non seppe il mezzo il giovanetto ardito / tener del raro e insolito viaggio, / ond'ebbe il nome sì famoso lito. // Così, madonna, chi del vostro raggio / s'infiamma il cor, alfin reman schernito, / se la ragion non segue sempre saggio», Rime CLx (cito dall'ed. a cura di M. Danzi, Modena, Panini, 1989, p. 196).

40. La seconda quartina riproduce infatti la situazione di Ovidio, Ars am. II, 94: «[...] quoque sub axe volas?», mentre l'ultimo verso quella di Ars am. II, $96:$ «[...] aequora nomen habent»; per il nome del v. I I, inoltre, vedi la nota I 5 . Evidenti, come sottolinea anche il commento di Danzi, le tracce petrarchesche: «celesti rai» (Rvf. 325, 99); «poggiare» nel significato di salire $(R v f .23,165)$

41. Appunto in Ovidio: «aequora nomen habent» (Ars am. II, 96): quel nome (cfr. v. I 2 del sonetto) che variamente risuona lungo tutta la tradizione icaria (vedi la nota I 5 ).

42. Velli, Un sonetto del Sannazaro cit., p. 6o-6r; cfr. Ovidio, Met. XII, 6i 6; Seneca, Herc. Oet. 1758 sgg.; Cesare, De bello civ. VIII, 788-89; Properzio, Eleg. II, 9, i 4 e, per il Sannazaro: Arcadia XI, I59 (poca fossa); Rime LIII, 75 (breve fossa); LXXXI, I4 (poca terra); LXxxIx, 2I (poca fossa); c, 96 (breve speco); Eleg. III, I, I 84 (brevis urna).

43. Il componimento reca come argomento Epitafio di Cesare: «Spargi di lauri, palme e mirti foglie, / viator, ché per qui intorno il spirto erra / di quel repente fólgore di guerra / che già de tutto el mondo adusse spoglie. // Questa urna angusta il ciner sacro accoglie, / ma el nome, qual in tomba non si serra, / tiene insepolto il ciel, l'acqua e la terra, / secur da tempo e da sue ingorde voglie. // Questi fu quel che con l'armata chioma / prima constrinse il mondo sotto il giogo / de l'alto imperio de l'antiqua Roma. // Oh felice sudor! poi che ogni luogo, / qual vinse avinto in la terrena soma, / occupa ancor dopo il funereo rogo!».

44. Di questo sonetto si ricorderà peraltro il Poliziano delle Stanze (I, 7), sempre rievocando la gara di emulazione con il modello omerico: «E se qua su la fama el ver rimbomba, / che 
la figlia di Leda, o sacro Achille, / poi che 'l corpo lasciasti intro la tomba, / lascia tacere un po' tuo maggior tromba / ch'i' fo squilllar per l'italiche ville, / e tempra tu la cetra a' nuovi carmi, / mentr'io canto l'amor di Iulio e l'armi».

45. Sannazaro, LV: «Quella c'a l'umil suon di Sorga nacque / et or sì chiara qui fra noi rimbomba, / levata a vuolo a guisa di colomba / sol per colui a cui tant'ella piacque, // quantunque in vile albergo occolta giacque / e stiasi or chiusa in una oscura tomba, / pur vive, per virtù di quella tromba / che per tal grazia al suo morir non tacque. // Tante donne leggiadre, oneste e belle, / e di stato maggior, son senza gloria, / e costei par c'ognor si rinovelle. // Beata lei, che 'n sì famosa istoria / lasciò 'l suo nome, ond'or su fra le stelle / risplende ornata d'immortal memoria!».

46. Vedi ad esempio Tansillo, Rime, son. 284 (a Garcilaso de Vega), in cui la serie (completa) appare nelle rime centrali delle quartine: «Se lieti ognor sen van Mincio ed Aufido, / u' la gran lira nacque e l'alta tromba, / ond' or Mecena più che mai rimbomba, / e la pietà di Enea, l'ardor di Dido; // se l'Arno e il Po bagnan superbi il lido, / qual per la cuna altrui, qual per la tomba; / s' il mar, dove, volando qual colomba, / Icaro cadde, ebbe onorato grido; // se sempre chiaro il Tebro entra nel mare / per tanti incliti spirti e figli suoi, / qual d'un leggiadro oprar, qual d'altro vago; // liete e superbe ed onorate e chiare / non per l'arene, Lasso, ma per voi, / mille e mill' anni andran l'onde del Tago»; e cfr. anche il sonetto 28 I, 9-I4, che riproduce la movenza sintattica di Sannazaro, LXxIx, 5-7, ma con le rime in -omba delle terzine (di cui riprende la struttura rimica CDC DCD): «Avventuroso più d'altro terreno, / Se con quel cigno uscia questa colomba, / ch'or t'ha di nuova gloria il grembo pieno. // Saria Mecena, che fra noi rimbomba, / Men noto; ella più chiara; e nel tuo seno, / Ov'egli ebbe la cuna, avria la tomba», LuigI TANsiLlo, Il canzoniere edito e inedito, a cura di E. Percopo, Napoli, Tip. Artigianelli, I926. Anche in un altro poeta di area meridionale, il De Jennaro, la successione rimica è volta a caratterizzare l'eco della fama terrena: «La fama tua, ch'al mondo ogi rispira / sì come altera e ben sonante tromba, / m'ha fatto veramente un uom di tomba, / bramando udire il suon de la tua lira. / Però che certo la virtù che spira / el cielo in te nel ciel chiara rimbomba, / se non mi inganna mia bianca colomba, / over colui ch'amando mi martira [...]», Pietro Jacopo De Jennaro, Rime e lettere, a cura di M. Corti, Bologna, Commissione per i testi di lingua, 1956, p. 67, sonetto Xxiv, I-8. Cfr. infine Britonio, Gelosia del sole: «Di me non fu più mortalmente afflitto / quello a cui fu interditto / come a me, lasso, il troppo alto alzarmi, / perchè non men vid'io precipitarmi / ch'egli, qualor solingo andando a volo, / apppresso sì del sol l'ardente lume / che le mal finte piume / mancandogli, con gran spavento e duolo / cadde nel mare; / or di lui fama e tromba, / che del suo nome ognor via più rimbomba», Gelosia del sole, Napoli, Sigismund Mayr, I 5 I 9, p. I64. Cfr. E. Raimondi, Il petrarchismo nell'Italia meridionale, in Atti del convegno internazionale sul tema Premarinismo e pregongorismo, Roma, Accademia Nazionale dei Lincei, I973, p. Iо9.

47. A cui si può aggiungere, in epoca quattrocentesca, un sonetto di Giovanni Aloisio tratto da El libro chiamato Naufragio del ms. cl. 3220* [Philol. I 94], canzoniere studiato da M. Santagata, La lirica aragonese: studi sulla poesia napoletana del secondo Quattrocento, Padova, Antenore, I979. Il componimento anticipa la strutturazione in una sezione "narrativa" (quartine) e di comparazione didascalica con l'io lirico (terzine) propria del sonetto del Tebaldeo, il tutto sotto il segno di un Petrarca anche troppo esemplare : «El miserello semplice figliuolo / de Dedalo, per l'arïa volando, / scordato del paterno pio comando, / lasciviendo già da vuolo in vuolo, // poggiò tant'alto su l'extremo polo, / el folle suo desir perseguitando, / onde 'l calor soi penne riscaldando, / cadde ne l'acque et pianse lì il suo duolo. // Quello Ycaro son io, che per salire / troppo alto, senza aver più legiere ale, / condocto ben da presso del morire, // nel pelago de lagrime et sospire / vegio affogarme, ove 'l pentir non vale, / né trovo più pietà del mio languire», Santagata, La lirica aragonese cit., p. I 94.

48. Riprende lo schema rimico del sonetto II il CII: «Quando, di ghiaccio armato, alzai tant'alto, / quanto poggiasse mai cosa mortale, / a la superba e bella impresa l'ale / del mio 
pensier, troppo animoso ed alto; // da duo begli occhi nel primier assalto / vinto rimasi; e, per maggior mio male, / m'accorsi tosto, ch'a chi troppo sale, / cadendo poi, tanto più noce il salto. // Arse le piume, io rovinoso a terra / cado, ove del mio ardir l'aspra memoria, / più che il presente danno, oggi m'attrista. // Ma non mi si potrà tor mai la gloria / d'aver impreso così nobil guerra, / ove, perdendo ancor, onor s'acquista».

49. Il sonetto II riproduce la cesura forte dell'incipit sannazariano (Icaro cadde qui: queste onde il sanno): «Amor m'impenna l'ale, e tanto in alto»; l'apparente ipotetica di Sannazaro Lxxix, 9-1 « «Ben pò di sua ruina esser contento, / se al ciel volando a guisa di colomba, / per troppo ardir fu esanimato e spento», Tansillo II, 7-8: «che, s'al superbo vol cadendo, io pero, / l'onor fia eterno, se mortal è il salto»; e tutta una serie di ulteriori tessere: «la ruina» (Sannazaro LXXIx, 9) «alta rovina» Tansillo III, I 2; l'«ardir» (Sannazaro LXxix, I I) «ardite penne» Tansillo II, I I; «la vita venne men, ma non l'ardire» (Sannazaro LXxIx, I4) «d'ardir colmo» Tansillo III, 4, «troppo ardimento» Tansillo III, I I; infine il «nome» (Sannazaro LXXIx, I3) «nome eterno» Tansillo II, io, per cui si veda anche Cartteo, Rime clxxxix, I-4: "Qualunque imprender vuol di simigliare / ai duo gran Toschi, ei simil par che sia / a cui, volando per troppo ardua via, / diede, cadendo, il nome al vitreo mare» (memore di Orazio, Carm. IV, 2, 3-4, cfr. la nota i 5 ).

50. Confronta le prove icarie di Sannazaro e Tansillo A. Afribo, Aspetti del petrarchismo di Luigi Tansillo, «Rivista di letteratura italiana», XII (1994), pp. 43-77; in particolare pp. 43-53, che registra anche, come volontario distanziamento del Tansillo rispetto al modello, l'uso insistito di figure della ripetizione, assenti o quasi nel Sannazaro: cfr. ad esempio, per il sonetto II, alto (I e 5), assalto (4); altero (6), salto (8); per il III: alte - altier (2); alta (I 2), alto (I 3). Nella citazione del sonetto sannazariano del saggio di Afribo si legga però esanimato e non, come compare alle pp. 45 e 48 , esaminato.

5I. E ancora Filenio GaLlo, Rime II, LIII, 5: «Chi vuol volar tropp’alto casca spesso» (cito dall'ed. a cura di M. A. Grignani, Firenze, Olschki, 1973).

52. Cfr. almeno Petrarca, Rvf. i4 I e Bembo, Rime, x, I 3-I 4. In Annibal Caro troveremo invece la commistione tra il motivo icario e quello della Fenice (condotta nei termini tuttavia caratteristici del motivo della farfalla) nella sestina Pellegrina fenice in mezzo un foco, 55-60: «Icaro già ne l'acqua, io ne la fiamma / lasserò del mio ardir memoria al mondo, / a l'alto mio sperar ben degno nido. / Che si dirà: costui sospinse il core / tanto verso una luce, che nel foco / strusse la cera, e 'ncenerio le piume».

53. Bernardo Tasso, Amori IV, 57: «Io pur m'inalzo con dedalee piume / per questo Ciel del vostro grande onore, / qual picciolo animal che per costume / vola a la luce ove s'incende e muore, // e temo che non strugga e non consume / la cera del desire il troppo ardore, / sì sono ardenti i rai del vostro lume, / ond'io caggia nel mar del proprio errore. // Deboli vanni certo a sì gran volo, / ma chi frena il desio vago di farsi / eterno in grembo de la vostra gloria? // dirassi almen da qui a mill'anni ch'arsi / le penne ardite per seguirvi solo, / e fia di me nel mondo alta memoria» (cito dall'ed. a cura di V. Martignone, Torino, RES, I995, II, 46). Si noti la varia rifrazione del sema ardor, in stretto rapporto col sannazariano ardir, il riferimento al mar del proprio errore (per cui si sì veda anche Salmi I, 20, I 5) sembra invece riportarci alle coordinate dottrinali dello pseudo-lucianeo De astrologia (cfr. nota 17 ). Un sonetto pressochè identico, se si esclude l'incipit e qualche piccola variante, troviamo nel componimento a nome di Onorato Fascitelli contenuto nelle Rime et versi in lode della Illustrissima et Eccellentissima Sigora Donna Giovanna Castriota Carrafa, duchessa di Nocera e marchesa di Civita Santo Angelo, Vico Equense, Cacchi, i 585 , p. 99, che, anche tenendo conto della flessibilità della categoria rinascimentale di imitatio, non si saprebbe come chiamare se non plagio (in corsivo le parti sottoposte a rifacimento): «Icaro io son, che con cerate piume / m'inalzo a sol del vostro grande onore, / qual semplice animal che per costume / vola a la luce ove s'incende e muore, // e temo che non strugga e non consume / la cera del desire el troppo ardore, / sì sono ardenti i rai del vostro lume, / onde caggia nel mar del 
proprio errore. // Debili vanni ho certo a sì gran volo, / ma chi frena el desio vago di farsi / eterno in grembo de la vostra gloria? // dirassi almen dopo mill'anni ch'arsi / le penne ardite per seguirvi solo, / e fia di me nel mondo alta memoria».

54. TAnsillo «S'un Icaro, un Fetonte / per troppo ardir già spenti il mondo esclama; / quel che perdêr di vita, elli han di fama / di me, farfalla pargoletta e frale, / qual fia la gloria tra' più vaghi augelli, / ch'ebbi ardir di spiegar le piccol'ale / al gran splendor de gli occhi e de' capelli, / ove Amor vinto regna, / e col volo cercai morte sì degna? / Qual pregio, udendo dire: / - Ogni farfalla, spenta in sul gioire / intorno a picciol lume morir suole; / quest'ebbe morte per gioir nel sole! -».

55. Cfr. M. Eigeldinger, Le mythe d'Icare dans la poésie française du XVTe siècle, "Cahiers de l'Association Internationale des Etudes Françaises», 25 (I973), pp. 26I-80; L. Sozzi, "Coeli cupidine tractus" cit.; J. G. Fucilla, Etapas en el desarrollo del mito de Icaro, en el Renacimiento y en el Siglo de Oro, "Hispanofila», 8 (1960), pp. I-34; J. H. Turner, The Myth of Icarus in Spanish Renaissance Poetry, London, Tamesis Books, 1976.

56. Mellin de Saint Gelais, Oeuvres complètes, Paris, Daffis, i873, I, pp. 299-300; cfr. M. Eigeldinger, Le mythe d'Icare cit., p. 263. Una nuova contaminazione tra il motivo icario e quello della fenice, in un contesto generale assai affine a quello del sonetto di Mellin di Saint Gelais, è in Joachim Du Bellay, Les amours, IV: « Si la beauté permettoit d'estre aymee / en si hault lieu, d'un tel coeur que le mien, / sans me vanter, dire j'oserois bien / qu'oncques beauté ne fut plus estimee: // non que le vol de ma plume animee / soit pour tenter un vol icarien, / mais vous louant elle ne craindroit rien, / si de faveur elle estoit emplumee. // Qui vouldroit donc un tel phoenix louër, / il vous fauldroit pour vostre l'advouër, / luy inspirant la force et le courage: // ou bien fauldroit qu'il teint le mesme ranc / de cest esprit, honneur de vostre sang, / qui fut nommé le Phoenix de son âge » (cito dall'ed. Oeuvres poétiques, a cura di H. Chamard e H. Weber, vol. 2, Paris, Didier, 1970, p. 237).

57. I sonetti II e III del Tansillo furono infatti pubblicati per la prima volta soltanto nel i 552 , nelle Rime di diversi illustri signori napoletani e d'altri nobilissimi intelletti. Nuovamente raccolte e con nova addizione ristampate. Terzo libro, Venezia, Gabriel Giolito de’ Ferrari.

58. Direttamente imitata nell'Ode au Seigneur des Essars, i 6 I-64 (Du Bellay, Oeuvres poétiques, vol. 4, Paris, Nizet, I 983 , p. I72): «Qui le Cygne dorien / le vol immiter desire / d'ung ozer icarien / se joint des ailes de cire»; cfr. anche Vers liriques, IV, I-8 (Du Bellay, Oeuvres poétiques, vol. III, Paris, Nizet, 1983, p. 97): «Quicunque soit qui s'estudie / en leur langue imiter les vieulx, / d'une enterprise trop hardie / il tente la voye des cieulx, // croyant en des ailes de cire, /dont Phebus le peult déplumer, / et semble, à le voir, qu'il desire / nouveaux noms donner à la mer ».

59. Du Bellay, Olive, cxv, 5-8. Nell'ed. a cura di E. Caldarini (Genève, Droz, 1974) viene indicato come modello di Du Bellay il sonetto di Petronio Barbati, compreso nel secondo volume giolitino delle Rime di diversi nobili uomini et eccellenti poeti nella lingua toscana. Libro secondo, Venezia, Gabriel Giolito de' Ferrari, I 547, Molza, il cui nome con si chiara tromba (che presenta la sequenza rimica tromba-rimbomba-colomba-tomba, di cui nelle precedenti pagine si è già abbozzata la fortuna): in effetti la mer te fust large tombeau si approssima più a «[...] Icaro, a cui diè il mar sì larga tomba» (v. 8 del sonetto del Barbati) che non a Sannazaro Lxxix, I 4: «Chi ebbe al mondo mai sì larga tomba?»; cfr. anche Montre le moy, qui te prise et t’honnore (v. I 2), che riprende «Ditelo a me, che v'amo, adoro e colo» (Barbati, v. I 2), e più in generale l'inflessione interrogativa, che nel Barbati occupa la parte centrale del componimento (seconda quartina e prima terzina), in Du Bellay tutto il sonetto ad eccezione dell'ultima terzina. Tuttavia ritengo che Du Bellay non potesse non avere in mente il ben più celebre sonetto sannazariano, soprattutto per la presenza del vol audacieux (v. 7), che rimanda alle «audaci penne» ivi menzionate al v. 2 (per il vol audacieux, sempre riferito a Ronsard, vedi 
Sonnets divers, xxxvIII, 6, in Ronsard, Oeuvres poétiques, II, p. 286). Ancora più al riparo da possibili cadute è il volo ispirato dalla grazia divina: cfr. La lyre chrestienne, in Oeuvres de l'invention de l'autheur, V, I66 (Ronsard, Oeuvres poétiques, IV, p. I44): "Que si tu veulx [Seigneur] luy prester l'aisle / alors d'ung vol audacieux / cryant ta louange immortelle, / je voleray jusques aux cieux».

6o. «Par une cheute subite / encor je n'ai fait nommer / du nom de Ronsard la mer / bien que Pindare j'imite» (Pierre de Ronsard, Oeuvres complètes, a cura di P. Laumonier, Paris, Hachette-Droz-Didier, I9I4-I967, I, p. I 18); cfr. anche Elegies xxxv (a Bartolomeo Del Bene), 45-48 (Ronsard, Oeuvres complètes, XVIII, pp. 254-55).

\section{I. RONSARD, Oeuvres complètes, XVII, p. 230.}

62. Come nella seconda parte del Bocage royal, il Discours à Monsieur de Cheverny, vv. 95 sgg (Ronsard, Oeuvres complètes, XVIII, pp. Ioo sgg): gli esempi di Icaro e Fetonte illustrano il destino di chi vuole «passer en grandeur le commun» (v. I I I), di chi in corte è divorato da una smodata brama di onori e grandezze.

63. Cfr. il sonetto de Les amours (I 5 52-53) Ce fol penser pour s'en voler plus hault: « Ce fol penser pour s'envoler plus hault, / apres le bien que haultain je desire / s'est emplumé d'ailles joinctes de cire, / propres à fondre aux raiz du premier chault. // Luy fait oyseau, dispost de sault en sault, / poursuit en vain l'object de son martire, / et toy, qui peux, et luy doys contredire, / tu le vois bien, Raison, et ne t'en chault. // Soubz la clarté d'une estoile si belle, / cesse, penser, de hazarder ton aisle, / ains que te voir en bruslant deplumer: // car pour estaindre une ardeur si cuizante, / l'eau de mes yeulx ne seroit suffisante, / ny suffisants toutz les flotz de la mer» (cito stavolta dall'ed. a cura di H. Weber e C. Weber, Paris, Garnier, I963, che segnala la derivazione). Sozzi, "Coeli cupidine tractus" cit., pp. I 808I, nota finemente come la versione di Ronsard orienti il sonetto verso un significato erotico più esplicito, a fronte di un ipotesto da questo punto di vista assai vago.

64. Come nota Sozzi, "Coeli cupidine tractus" cit., p. I9I, il coup de foudre per Astrée dovrebbe precedere, e non seguire, il volo della passione.

65. Rimando per la fitta bibliografia al recente contributo di R. Gorris, Desportes et les Imitations de l'Arioste, in AA.vv., Philippe Desportes (I546-1606). Un poète presque parfait entre Renaissance et Classicisme, a cura di J. Balsamo, Paris, Klincksieck, 2000, pp. I73-2I I; in particolare $177 \mathrm{n}-78 \mathrm{n}$.

66. Ad esempio Les amours de Diane XXVIII e Procez contre Amour au siège de la Raison, 67-68 (cito dall'ed. a cura di V. E. Graham, Genève, Droz, I959, pp. 67 e I 56); Élégies VI, 57-60 (che accosta la figura Icaro a quella dei Giganti in rivolta; cito dall'ed. a cura di V. E. Graham, Genève, Droz, I961, pp. 50-51); cfr. Eigeldinger, Le mythe d'Icare cit., pp. 274 sgg.; Sozzi, "Coeli cupidine tractus" cit., pp. I96 sgg. Più interessante il sonetto II della Cléonice (in particolare i VV. I-II), che mostra la sua derivazione dalla trafila del componimento ariostesco Del mio pensier, che cosi veggio audace (da cui dipende anche il modello citato dal Graham, il sonetto di Girolamo Acquaviva Io dissi al mio sperar, ben guiderai, compreso nelle Rime di diversi signori napoletani. Libro settimo, Venezia, Gabriel Giolito de' Ferrari e fratelli, I 5 5), non senza un tocco di Sannazaro e Tansillo (vedi i particolari dell'onde, della tumbe per il primo, e per il secondo del nouveau sentier, che riprende il novo assalto di II, 4): "J'ay dit à mon Désir: - Pense à te bien guider, / rien trop bas, ou trop hault, ne te face distraire -: / il ne m'escouta point, mais jeune et volontaire, / par un nouveau sentier se voulut hazarder. // Je vey le ciel sur luy mille orages darder, / je le vey traversé de flamme ardente et claire, // se plaindre en trebuchant de son vol temeraire, / que mon sage conseil n'avoit sceu retarder. // Apres ton precipice, ô Desir miserable! / Je t'ay fait dedans l'onde une tumbe honorable / de ces pleurs que mes yeux font couler jour et nuit ». 
67. Degno di nota che «giovane audace» è chiamato Fetonte nel volgarizzamento ovidiano di Gabriele Simeoni, letterato attivo alla corte di Francia (Vita et Metamorfoseo d'Ovidio. Figurato et abbreviato in forma d'epigrammi, Lione, Giovanni di Torne, I 559 , p. 36).

68. Segnalata anche dall'editore V. E. Graham (Genève, Droz, I960, p. I I).

69. Di qui la tendenza alla sintesi: vedi ad esempio il v. I 3 : «le ciel fut son desir, la mer sa sepulture» che riunisce le componenti sparse nel sonetto di Sannazaro LXXIX ai vv. io «se al ciel volando» e I 3 «un mar sì spazioso».

70. Per cui vedi il sonetto della Cléonice citato alla n. 66.

71. Come ha notato Sozzi, "Coeli cupidine tractus" cit., p. I95.

72. Nel sonetto IX de Les amours d'Hippolyte, 5-I4 (ed. cit., p. 20), si sottolinea infine l'ineluttabilità di tale parabola: «Je voy bien mon erreur, et que j’ai commencé / (nouveau frere d'Icare) un vol trop temeraire, / mais je le voy trop tard, et ne m'en puis distraire, / par la mort seulement il peut estre laissé. // Raison, arriere donc: ta remonstrance est vaine, / si je meurs en chemin je serai hors de paine, / et par mon haut desir j’honore mon trespas. // Il faut continuer, quoy que j'en doive attendre: / ce fut temerité de l'oser entreprendre, / ce seroit lascheté de ne poursuivre pas ».

73. Prova ne sia l'insistenza e la pregnanza con cui ricorre il termine caduta nelle Lettere: «et andava rivolgendo fra me stesso, che, s'in mediocre stato che pendeva a l'umiltà io era stato così fieramente soggetto a gli strali de l'invidia cortegiana, maggiormente sarei sottoposto a i medesimi, se dopo così gran caduta, con subito ed inaspettato rivolgimento di fortuna, io passassi da l'un a l'altro estremo di favore e di condizione» (al Duca d'Urbino Francesco Maria Della Rovere, s.d. [ma I 578 ], Torquato TAsso, Lettere, a cura di C. Guasti, Firenze, Le Monnier, I 8 54, I p. 280 n. I09); «Ora dopo cinque anni d'infemità e di travagli, se per pazzia son caduto dal mio grado, come dicono, la pazzia è anzi degna di compassione che di pena» (al card. Giovangirolamo Albano del 23 maggio I 58 I, TAsso, Lettere, II, p. I 26 n. I62); «[...] perch'io son già caduto e ruinato, e molt'anni sono ch'io tento di risorgere invano ne l'opinione de gli uomini e di ristorarmi con la grazia de' principi» (a Scipione Gonzaga dell' I ottobre I 587 , TASso, Lettere, III, p. 262 n. 899). Ci riporta ad un contesto affine l'allusione del trattato Il secretario: «Ma coloro ch'essendo nuovi ne la corte, sono privi di tutti gli altri appoggi, et ascendono da se medesimi, di leggieri possono cadere o esser gittati a terra; però deono schivar ogni superbia et ogni soverchio ardire, essendo la caduta tanto più vergognosa, quanto è da luogo più riguardevole», Il secretario del sig. Torquato Tasso, diviso in due parti. Con alcune rime nove del medesimo, Ferrara, Vittorio Baldini, I 587, p. 45; così pure, infine, Rime 848, 9-I 4: «Ma io, che dopo te discesi in questo / spazio di vita incerta, al fin del corso / non so s'io sia vicino anco o lontano; // e lasso e fral per la caduta e mesto / ti seguo; e se dal ciel non ho soccorso, / a' premi corro de' pentiti in vano» (a Giulio Mosti, carceriere del S. Anna; cito da Torquato Tasso, Rime, a cura di B. Basile, Roma, Salerno, 1994, che riproduce il testo dell'ed. Solerti).

74. Sul quale si veda la breve ma documentata nota di M. Colaninno, Gli echi del precipizio. Il mito di Fetonte nelle "Rime" del Tasso, «Studi tassiani», XLIV (1996), pp. I 3 5-46.

75. Tale, ad esempio, quello che lega il volo icario alla fama: cfr. il secondo sonetto al principe di Conca, Tasso, Rime I 470: «Oltre il Gange, oltre il Nilo e l'Indo e 'l Reno / volar devrian con più sonanti carmi / la vostra gloria e l'alte imprese e l'armi, / e i gran nomi a cui 'l tempo è gran veneno; // né potrebbe una man vergarne appieno / le carte, né scolpirne i bianchi marmi, / né cantarne una lingua, e io temo alzarmi / sovra le nubi, e 'n grembo al mar Tirreno, // quasi Icaro, lasciar l'ardite piume. / Dunque altrui più le sparga, e, come giusto, / passi la fama vostra Abila e Calpe: // da me ristretta infra due mari e l'Alpe / parria tra monti ripercosso lume / o vento che rimbombi in loco angusto». 
76. Ma per le «ardite piume» vedi il sonetto I 470 citato alla nota precedente. Ritroveremo le «ardite piume» come sintagma incipitario in un sonetto icario di un antico compagno di strada del Tasso, Ascanio Pignatelli, membro dell'Accademia degli Eterei; cfr. Rime XVIII: «L'ardite piume, onde 'l mio cor si cinse, / furo, o Donna, i pensieri, et a la face / d'un bel guardo volando Icaro audace / folle di me credenza a voi mi spinse; // ivi sperando amando arsi, ma vinse / mia debil forza il forte ardor vivace, / e del mio pianto un ampio mar vorace / la speme ben, ma non le fiamme estinse: // ahi ch'esca fur le lagrime, e 'l dolore / di maggior foco, onde qual secco in herba / fior le speranze incenerì l'ardore, // che 'l vostro lume ascoso incendio serba / ch'arde non visto, e da i dolci occhi fore / dolce trapassa amara voglia acerba», cfr. Rime del sig. Ascanio Pignatello caualiero napoletano. Date nuouamente alle stampe da Gio. Battista Crispo da Gallipoli, Napoli, Stigliola, per Gio. Tomaso Todino, I 593, p. 9.

77. Il Messaggiero (redazione definitiva), I73-174: «E or rammentati di quel c'hai letto leggendo Omero, qundo Giove dice che, s'egli mandasse giù una catena dal cielo sin a la terra e tutti gli dei cercassero, apprendendosi a quella catena, di tirar Giove a sé, non potrebbono, ma egli di leggieri a sé tutti gli tirarebbe. Questa catena altro non significa che la catena amorosa, con la quale Iddio potentissimo non è mosso dagli dei minori o da l'altre creature, ma egli tutte le muove come amato e desiderato», Torquato Tasso, Dialoghi, a cura di B. Basile, Milano, Mursia, I991, p. 83.

78. Torquato Tasso, Gerusalemme liberata IV, IX: «Tartarei numi, di seder più degni / là sovra il sole, ond'è l'origin vostra, / che meco già da i più felici regni / spinse il gran caso in questa orribil chiostra, / gli antichi e altrui sospetti e i feri sdegni / noti son troppo, e l'alta impresa nostra; / or Colui regge a suo voler le stelle, / e noi siam giudicate alme rubelle»; Gerusalemme liberata XV, XXVI, I-6: «Ei passò le Colonne, e per l'aperto / mare spiegò de' remi il volo audace; / ma non giovogli esser ne l'onde esperto, / perché inghiottillo l'oceàn vorace, / e giacque co 'l suo corpo anco coperto / il suo gran caso, ch'or tra voi si tace». Si noterà poi che «alta impresa» di Gerusalemme liberata IV, IX, 6 si ritrova nelle citate Rime 794, 9 e, al plurale, Rime I $470,3$.

79. TAsso, Rime 738: «Aspirava, signor, novo Fetonte / a gir sul carro de la luce adorno / de la mia gloria ed a portare il giorno / per l'alte vie del cielo a me non conte, // quando ecco vidi fulminar la fronte / di Giove irato e 'l ciel turbarsi intorno, / e fulminato caddi e nel mio scorno, / lasso!, non mi celò fiume né fonte, // non mi pianse sorella: aspro e maligno / caso! Ma pur ho voce anco e parole, / onde mi lagni in loco imo e palustre; // e forse fia che, tua mercede, in cigno / alfin mi volga e 'l tuo gran volo illustre / segua così da lunge inverso il sole».

80. Torquato Tasso, Rime [...]. Di nuovo datte [sic] in luce, con gli argomenti et esposizioni dello stesso autore, Brescia, Pietro Maria Marchetti, I 593, Parte prima, p. 7.

8I. TAsso, Rime 668, 6i-65: « onde, se ben del canto / forse m'appago e vanto, / temo, cigno infelice, i fulmin tuoi; / e sol pronte le penne / colà saran dove il tuo ciglio accenne». Cfr. anche l'espressione «cigno in mia prigion» di Rime I 28, 5, per cui si veda l'autocommento al v. 5, dove il Tasso precisa: «I Cigni non sogliono tenersi in gabbia: però dimostra la sua infelicità maggiore», TAsso, Rime [...] con gli argomenti $[. .$.$] , p. I 46$.

82. Così Antonio Costantini che, in uno dei sonetti composti per la morte del poeta, ritrae il suo volo sublime negli spazi celesti, accennando alla sua esemplare esistenza terrena attraverso l'immagine sannazariana della caduta di Icaro ("gran caso): «Torquato, or sì che nel celeste regno / contempli il sole ardente, e l'auree stelle, / e forme anco di lor più vere, e belle / dove a gran pena vola umano ingegno; // e fiamme e lampii, ove con fero sdegno / Giove accoglie le nubi e le procelle, / e tonando spaventa alme rubelle, / ch'è di tempesta altrui presagio e segno. // E se rivolgi de l'altera mente / a le cose terrene il presto volo, / 
formi nel tuo gran caso illustre essemplo, $/ /$ in cui la tua virtù miro e contemplo, / che te stesso formasti, o primo, o solo / spirto immortal d'eterna gloria ardente» (il sonetto si trova nella sezione dedicatoria di ToRQuato TAsso, Lettere [...] non più stampate Bologna, Presso Bartolomeo Cochi, I6I6). Una esplicita chiave di lettura icaria per la vicenda umana del Tasso è invece proposta da Bartolomeo del Bene nell'ode $A$ Torquato Tasso. Poeta e filosofo rarissimo del secol nostro, nella quale, mostrando l'autore «di quanta cecitade o impietade sia pieno l'uomo, che per le cose visibili e mobili non crede e non conosce le invisibili e immobili, cioè le divine», è affrontata una problematica moraleggiante. Al termine del componimento l'autore si rivolge a Tasso domandando le ragioni della sua caduta: «Chi t'ha, Torquato mio, / sospinto con immagini sì false / la mente, che di Pindo al sommo salse, / in antro di furor sì tetro e rio? // Arebbe Amore scorto / con finte larve ogni tuo senso ardente, / dove solo dovea poggiar la mente, / onde cadesti tu spennato e smorto, // qual Icaro novello, / per troppo ardir, per troppo caldo et lume, / per che, qual l'ebbe il mar, del Po 'l gran fiume / dal tuo caso aggia nome eterno e bello?», Bartolomeo Del Bene, Odi XXVIII, edite da G. Carducci e S. Ferrari, Bologna, Zanichelli, I 908, pp. 67-68. L'ode venne composta nel i 584 . Devo questi riscontri, e quelli che seguono, al volume di A. Coppo, Allombra di malinconia. Il Tasso lungo la sua fama, Firenze, Le Lettere, 1997, pp. 79 sgg.

83. Cfr. il sonetto di Claudio Achillini Quel dolce cigno, ch'atterò l'impero: «Quel dolce cigno, ch'atterò l'impero, / che 'l gran cigno di Sorga aveva, or tristo, / cantando in suono all'or confuso e misto / d'affetto, di stupor, dolce, severo; // quel cigno (il dirò pur) che vinse Omero, / stampando in tante perle il sacro acquisto, / che fece l'alto cavalier di Cristo / togliendo la gran tomba al Trace altero; // quel, Segni mio, che tante volte e tante / volle teco albergare, amico fido, / di tuoi costumi e di tue muse amante, / ch'al fin volgesse a Cinzio il core e i carmi, / fondando a le virtù la patria e 'l nido, / onor di Cinzio incomparabil parmi» (Tempio allillustrissimo et reverendissimo signor Cintio Aldobrandini cardinale S. Giorgio nipote del Sommo Pontefice Clemente ottavo, Bologna, presso gli eredi di Giovanni Rossi, I600, p. 95); infine un sonetto di Angelo Grillo in cui si rivelano esplicitamente i contrassegni della tradizione icaria (il nome, la rima rimbomba-tromba) : «Cigno sublime a la prigion famosa, / dov'il tuo mandi e gli altri nomi a volo, / con l'ali del pensier sovente volo, / e dove Apollo ha maggior pregio o posa? // Ivi, qual ti vid'io tra la noiosa / schiera de' pensier tuoi colmo di duolo, / tal or ti veggio e parlo e inalzo e colo, / s'io leggo alto tuo carme o dotta prosa; // et in leggendo dico: o mostro, o lume / di nostra età, chi ti pareggia o appressa? / O qual nome più chiaro oggi rimbomba? // Ma, dhe, com'oso, o mio terrestre nume, / cantar di te? Grillo notturno, ah, cessa, / che degna è sol di lui l'alta sua tromba», Angelo Grillo, Parte prima delle rime [...] nuovamente date in luce, in Bergamo, appresso Comino Ventura, I590, p. 74v, son. cLXVI delle Rime morali. 